\title{
Platooning With IVC-Enabled Autonomous Vehicles: Strategies to Mitigate Communication Delays, Improve Safety and Traffic Flow
}

\author{
Pedro Fernandes, Member, IEEE, and Urbano Nunes, Senior Member, IEEE
}

\begin{abstract}
Intraplatoon information management strategies for dealing with safe and stable operation are proposed in this paper. New algorithms to mitigate communication delays are presented, and Matlab/Simulink-based simulation results are reported. We argue that using anticipatory information from both the platoon's leader and the followers significantly impacts platoon string stability. The obtained simulation results suggest that the effects of communication delays may be almost completely canceled out. The platoon presents a very stable behavior, even when subjected to strong acceleration patterns. When the communication channel is subjected to a strong load, proper algorithms may be selected, lowering network load and maintaining string stability. Upon emergency occurrences, the platoon's timely response may be ensured by dynamically increasing the weight of the platoons' leaders data over the behavior of their followers. The simulation results suggest that the algorithms are robust under several demanding scenarios. To assess if current intervehicle communication technology can cope with the proposed information-updating schemes, research into its operation was conducted through a network simulator.
\end{abstract}

Index Terms-Autonomous vehicles, intervehicle communication (IVC), network simulation, platooning, traffic information management, traffic simulation.

\section{INTRODUCTION}

$\mathbf{U}$ RBAN traffic congestion is becoming an unmanageable problem. Its consequences span from economics, public health, and energy consumption to pollution, among others. Traffic regulation through dynamic tolls may temporarily mitigate its impact, but new long-term solutions are required.

As concerns urban transportation, it seems likely that demand tends to always surpass supply. Nevertheless, mobility of urban inhabitants and goods should be granted and improved. However, more road network construction is not always viable and might not even ensure sound benefits. The problem to tackle resides on how to improve road capacity while reducing both travel time and traffic congestion. Platooning may help

Manuscript received March 10, 2011; revised June 30, 2011 and September 24, 2011; accepted December 3, 2011. Date of publication January 20, 2012; date of current version March 5, 2012. This work was supported by Portuguese Foundation for Science and Technology under Grant PTDC/SEN-TRA/099413/2008. The work of P. Fernandes was supported by the Portuguese Foundation for Science and Technology through Research Fellowship SSFRH/BD/38605/2007. The Associate Editor for this paper was L. Vlacic.

The authors are with the Institute of Systems and Robotics, Department of Electrical and Computer Engineering, University of Coimbra, 3030-290 Coimbra, Portugal (e-mail: pedro@ isr.uc.pt; urbano@isr.uc.pt).

Color versions of one or more of the figures in this paper are available online at http://ieeexplore.ieee.org.

Digital Object Identifier 10.1109/TITS.2011.2179936 to improve lane capacity, if constant spacing is used [1]-[3]. However, to be effective with respect to traffic flow, platooning should be performed with vehicles evolving on dedicated tracks and operating on a nonstop basis from origin to destination [4]. As such, by eliminating the stop-and-go problem of common car and transit systems, platooning could contribute to a faster and more comfortable mobility with higher energy efficiency.

Intervehicle communication (IVC) is emerging as a prominent technology for helping in reducing traffic congestion. Recent technological advances on communications, such as dedicated short-range communications (DSRC) [5]-[7] and long-term evolution advanced (LTE-Advanced) [8], as well as on cooperative adaptive cruise control (CACC) [9], personal rapid transit (PRT) [4], and autonomous vehicles, such as cybercars [10], are opening new research avenues that might show the usefulness of platooning in urban mobility improvement, when applied to automated vehicles. Moreover, since the vehicles in platooning are expected to be allowed to move very close to each other, a considerable reduction on energy consumption might be possible [4], once each follower vehicle would benefit from a reduced air drag due to their precedent vehicle.

In the present stage of our research, some assumptions are made. First, only longitudinal control of vehicles is assumed. Second, we consider only autonomous vehicles platooning. Third, we consider a platoon dimension up to $31 \mathrm{~m}$ long (a maximum of eight vehicles $3 \mathrm{~m}$ long and $1 \mathrm{~m}$ apart). Next, line-of-sight (LOS) communication between each leader and its followers is assumed. Then, our communication algorithm uses time slots, aiming to avoid intraplatoon packet collisions. Finally, the vehicles' dynamics are considered identical. These assumptions aim to isolate the effects of communication delays on the platoon's behavior. However, they must be assessed to find if they are acceptable with respect to communication constraints. For that purpose, the NS-3 [11] network simulator is used in testing several demanding platoon scenarios.

Among others, the following problem can be formulated: How can platoon stability be ensured under the negative effects of communication delays? Control methods for ensuring platoon string stability exist [3], however, under the assumption of the use of IVC without delays. This is not a realistic assumption, and communication delays are known to create hardly manageable string instability [12]. Intraplatoon information management strategies to deal with this problem are proposed in this paper. Our main goal is to ensure platoon string stability under the unavoidable presence of communication delays. To achieve 


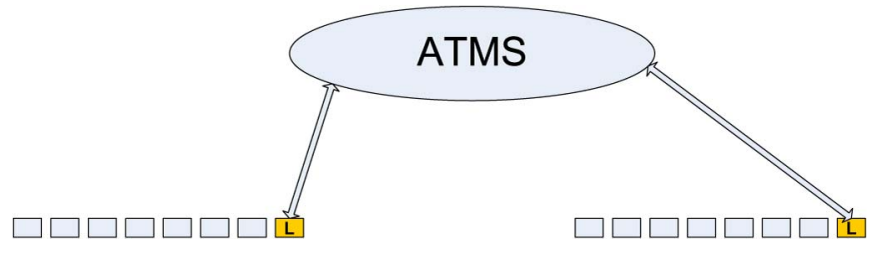

Fig. 1. Hierarchical ATMS (partial view).

our goal, we aim to enclose the communication, computation, and actuation delays within an upper bound delay, which is managed at a higher level in a way that presents no significant effect on platoon string stability.

An appropriate scenario for a platoon-based mobility system consists of its implementation with autonomous electric vehicles (EVs) in a network of dedicated tracks with offline stations and nonstop trips from origin to destination, managed by a hierarchical multilayered advanced traffic management system (ATMS) [13], [14]. As such, traffic of conventional vehicles is not considered in this scenario. Fig. 1 shows a partial view of the proposed ATMS. This study focuses on the appropriate operation of each platoon, dealing with its string stability when facing communication delays. Nevertheless, this work is part of a more vast research, where other important problems are also the subject of study, such as the rules to construct a platoon and leaders' election. For a more detailed description, see [13] and [14]. A brief summary of those rules follows: All leaders are elected by an upper level of the ATMS, receiving from it their acceleration patterns in predetermined time periods; a vehicle is only allowed to enter the track to evolve isolated, if it will occupy a leader's vacant location at precise positioning, and it will perform as leader; a set of up to eight vehicles may be admitted to the track, despite not joining other vehicles evolving in the track, as long as the first vehicle occupies a leader's vacant position and performs as leader, whereas the remaining vehicles perform as followers, under the control law (6) of Section IV; an isolated vehicle, or a set of vehicles, may enter the track just behind other evolving vehicles, performing as follower(s), if the total number of vehicles is $\leq 8$; when a leader leaves the track, the lead vehicle of the platoon's remaining vehicles must assume leadership; should some unforeseeable event occur, leaders are allowed to perform emergency stops, under the information-updating algorithms described in Section VI.

The upper levels of the proposed ATMS should control the whole system, including platoon leaders' appropriate operation and admission of vehicles into the system, to avoid congestion, even when operating close to its maximum capacity. The management of free vehicles to deal with asymmetrical transportation demand should also be ensured by the ATMS.

Since communication failures are of considerable importance in such scenarios, we further explore communication algorithms that might ensure that communication errors do not occur or are minimized. For that purpose, network simulations were performed with NS-3, and results are reported.

In the succceeding sections, we present the applied platoon control models, communication and information-updating schemes, and simulation models and results.
TABLE I

FIELDS OF KNOWLEDGE TO CONSIDER

\begin{tabular}{ll}
\hline \hline Field & Some relevant topics \\
\hline Traffic & Theory, fundamental diagram, traffic flow \\
Communications & DSRC/WAVE, LTE-Advanced (4G) \\
Simulation & Traffic and communication networks \\
Control & Constant spacing platooning, string stability \\
Autonomous vehicles & Communicant, cooperative \\
Computer Science & C++, Python, Java, application architecture \\
Energy & Consumption, efficiency, EV's \\
\hline
\end{tabular}

\section{RELATED WORK}

This research encompasses several fields of knowledge (as shown in Table I). A great number of research studies and results have been published in the last years, concerning the aforementioned fields. Many of them are related with autonomous vehicles [15], adaptive cruise control [16], CACC [9], cooperative active safety system [17], among other research topics.

In [18], Shladover reviewed the history of the founding of the California Partners for Advanced Transit and Highways (PATH) program and of the national intelligent transportation system program in the U.S., providing perspective on the changes that have occurred during the past 20 years. In [19], Varaiya discussed key issues related to a highly automated intelligent vehicle and highway system (IVHS) and proposed an IVHS control system architecture. Swaroop et al. [1], [2] investigated various platooning control strategies. Alvarez and Horowitz [20] researched the conditions to achieve safe platooning under a normal mode of operation. Horowitz and Varaiya described in [21] a five-layer automated highway system control architecture, involving the infrastructure and the vehicles. Rajamani et al. [22] developed a controller of platoons with constant spacing, concluding that autonomous control is not enough to ensure string stability. He found that only with IVC is the string stability of vehicle platoons achieved. The method was experimentally evaluated in field tests. Kato et al. [23] described the technologies of the cooperative driving with automated vehicles and IVCs in the Demo 2000 cooperative driving. Global control strategies for vehicle platooning systems are addressed in [24]. Recently [25], Broggi's team completed a 3-month journey of $13000 \mathrm{~km}$, with four autonomous EVs equipped to drive in leader-follower configuration.

The effects of communication delay on the string stability of platoons were researched by Mahal [12]. The main conclusion was that the string stability of platoons is compromised in the presence of communication delays. A simple method to deal with string instability due to communication delays was proposed in [26]. The solution consisted of a simultaneous update of all vehicles' controllers with the delayed information. However, it did not deal with anticipatory information from the leaders or the followers.

PRT experimental testing and field applications have been reported [27]. However, the benefits of such novel transportation system with regard to light rail or conventional cars are yet to be clearly demonstrated. Simulators more focused on PRT systems 
have been proposed [28], [29]. However, it is not clear how they model both communications and cooperation.

In [30], we addressed the implementation of IVC-enabled autonomous vehicle platooning capabilities in the Simulation of Urban MObility (SUMO) traffic simulator [31]. Dold and Stursberg [32] proposed an approach to robust model predictive control for distributed systems with chain structure, which includes a scheme for communicating predicted control trajectories between the subsystems. Wang and Pham [33] developed a high-fidelity cosimulation platform to study the motion and control of a vehicle platooning system, in MSC ADAMS and Matlab/Simulink.

Safety-based IVC is crucial for appropriate platooning operation. However, DSRC broadcasting performance does not present enough reliability to ensure safe vehicle operation in such scenarios. Several proposals have recently been made, aiming to improve its features. In this context, Yu and Biswas [34] presented a novel medium access control (MAC) protocol for IVC using DSRC, which consists of a self-configuring timedivision multiple-access protocol with short and deterministic delay bound capabilities. Torrent-Moreno et al. [35] proposed a distributed transmit power control method to control the load of periodic messages on the channel. Bi et al. [36] presented a cross-layer broadcast protocol to allow efficient and reliable message dissemination in IVC systems. Palazzi et al. [37] proposed a novel IVC architecture that adapts its functionalities to efficiently serve applications by quickly propagating their messages over a vehicular network. Tabatabaei et al. [38] presented an improvement on simpler propagation models for simulations by augmenting ray-tracing-derived models of wireless propagation. However, there are few approaches concerning the analysis of DSRC use in a constant spacing platooning environment, presenting specific problems with which to deal.

\section{TRAFFIC FLOW LIMITS}

Maintaining a desired intervehicle spacing in platoons demands a tight control of each vehicle. In case of human-driven vehicles, intervehicle spacing is defined through a constant time headway, which means that increasing speed implies increased spacing. According to [39], the "desired minimum gap" $s^{*}$ between two vehicles under the intelligent driver model (IDM) [40] is given by

$$
s^{*}(v, \Delta v)=s_{0}+v T+\frac{v \Delta v}{2 \sqrt{a b}}
$$

with $\Delta v:=v_{\alpha}-v_{\alpha-1}$, where $\alpha$ denotes the follower vehicle and $\alpha-1$ denotes the leading vehicle, $s_{0}$ is the minimum distance in congested traffic, $a$ is the maximum acceleration, and $b$ is the "comfortable deceleration." The last term of (1) is only significant in nonstationary traffic, when $\Delta v \neq 0$. Term $v T$ is the most relevant one to the resultant spacing in stationary traffic, where $T$ denotes the "safety time gap." When speed increases, $s^{*}$ proportionally increases, which means that the road vehicle density decreases. With human drivers, it is not possible to avoid this performance pattern, even with IVC, because human reaction time is the main reason for the need of the safety gap proportional to the vehicle speed. That reaction
TABLE II

LANE CAPACITY USING Platoons

\begin{tabular}{|c|c|c|c|c|c|c|}
\hline $\mathbf{v}$ & $\mathbf{n}$ & d (m) & D (m) & C (ve/h) & SUMO & k (veh/km) \\
\hline \multirow{5}{*}{ 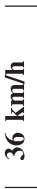 } & 1 & - & 15 & 2000 & 2400 & 55 \\
\hline & 5 & 1 & 30 & 3674 & 3675 & 102 \\
\hline & 8 & 1 & 30 & 4721 & 4730 & 131 \\
\hline & 15 & 1 & 30 & 6067 & 6070 & 169 \\
\hline & 20 & 1 & 30 & 6606 & 6600 & 183 \\
\hline \multirow{5}{*}{$\frac{\underset{D}{D}}{N}$} & 1 & - & 25 & 2571 & 2800 & $\overline{36}$ \\
\hline & 5 & 1 & 30 & 7347 & 7340 & 102 \\
\hline & 8 & 1 & 30 & 9443 & 9450 & 131 \\
\hline & 15 & 1 & 30 & 12135 & 12140 & 169 \\
\hline & 20 & 1 & 30 & 13211 & 13210 & 183 \\
\hline
\end{tabular}

time of about $1 \mathrm{~s}$ leads to the lack of string stability in vehicle platooning. This means that, if the lead vehicle decelerates, the spacing between vehicles may decrease toward the end of the platoon, leading to possible collisions.

According to [19], road capacity may be increased by using platoons with tightly spaced vehicles. The formulation to determine road capacity is given as follows:

$$
C=v \frac{n}{n s+(n-1) d+D}
$$

where $C$ represents the number of vehicles (in vehicles per second), $d$ is the intraplatoon spacing (in meters), $D$ is the interplatoon spacing $(m), s$ is the vehicle length (in meters), $v$ is the steady-state speed (in meters per second), and $n$ is the number of cars in each platoon.

Based on (2), Table II presents lane capacity values for $s=$ $3 \mathrm{~m}$, and velocities of 36 and $72 \mathrm{~km} / \mathrm{h}$. Parameter $k$ represents the vehicle density in vehicles per kilometers. The spacing of free vehicles $(n=1)$ is consistent with the minimum gap obtained from the IDM model (1), in which human drivers, presenting reaction times of approximately $1 \mathrm{~s}$, need about $15 \mathrm{~m}$ of safety gap at a speed of $36 \mathrm{~km} / \mathrm{h}$. Note the significant improvement of lane capacity with platoons of eight vehicles each, when compared with the free vehicle case, even with an interplatoon spacing of $30 \mathrm{~m}$, which is double the spacing of individual vehicles $(15 \mathrm{~m})$. It is also worth mentioning, that, for the free vehicle case, the values were obtained under ideal conditions, i.e., without traffic lights, intersections, or other obstacles usually present in an urban environment. Doubling the velocity in Table II leads to a slight improvement of the lane capacity $(28.5 \%)$ in the case of free vehicles. However, the capacity doubles with platoons of eight vehicles, when compared with the analogous case at the speed of $36 \mathrm{~km} / \mathrm{h}$, resulting in more than three times the capacity of the lane with free vehicles, with the same speed considered. Table II also presents the traffic flow results of simulations of constant speed platoons, performed on the SUMO traffic simulator. In Table II, SUMO results for $n=1$ were obtained using the Krauß carfollowing model [41]. The platooning results $(n \geq 5)$ were obtained with the modified SUMO reported in our previous work [30], which consists in a novel car-following implementation allowing to simulate platooning of autonomous vehicles with constant spacing. The simulation scenario consisted of a several-kilometers-long straight lane, where a large sequence 


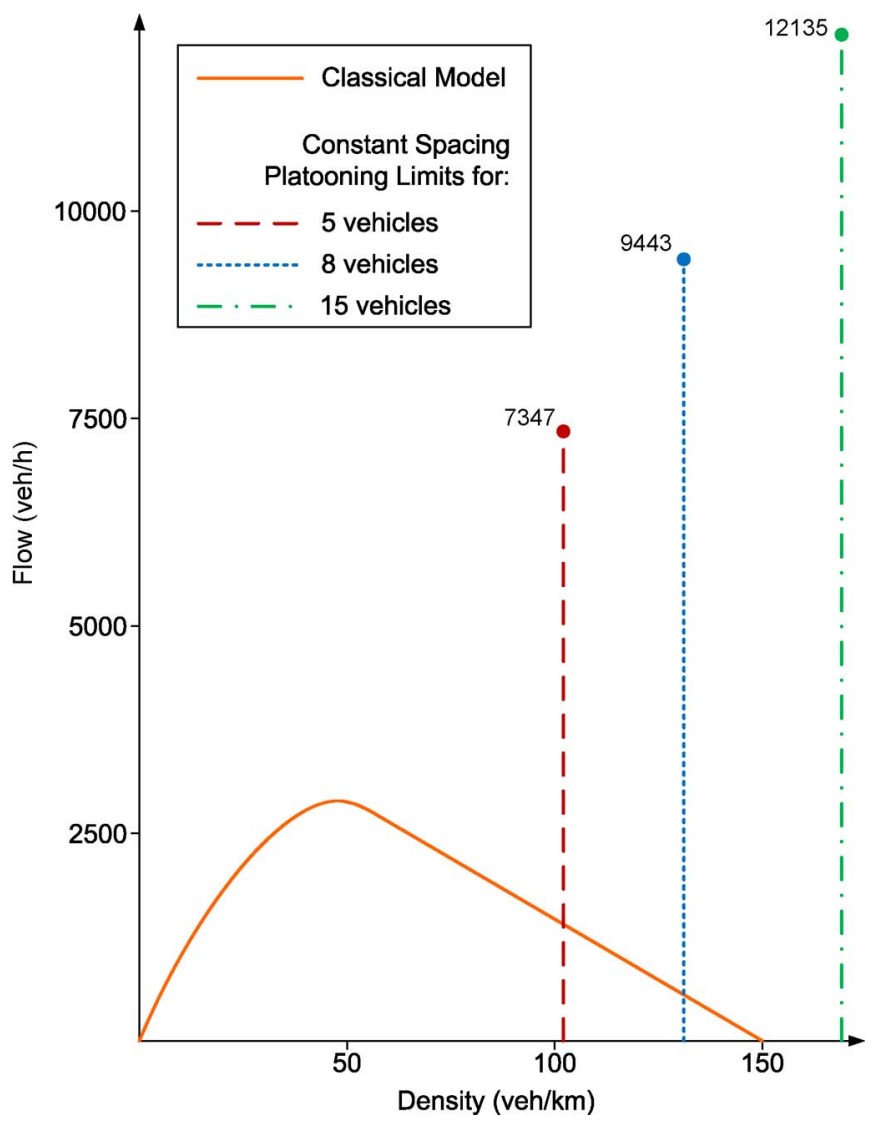

Fig. 2. Fundamental diagram of traffic flow. Limits for platoons of five, eight, and 15 vehicles each, separated by $30 \mathrm{~m}$, with vehicles $3 \mathrm{~m}$ long and $1 \mathrm{~m}$ apart from each other, are depicted in dashed lines, with upper values for the speed of $72 \mathrm{~km} / \mathrm{h}$.

of platoons evolved in formation. At some defined points of the lane, E1-type loop detectors were installed in SUMO, allowing to obtain the aggregate results of traffic flow. Although slightly higher in the case of free vehicles, these results are consistent with those theoretically drawn from (2).

In Fig. 2, we represent the fundamental diagram of traffic flow [42]. By the fundamental relation of traffic flow theory

$$
q=u \times k
$$

where $q$ represents traffic flow (in vehicles per hour), $u$ the vehicle speed (in kilometers per hour), and $k$ the vehicle density (in vehicles per kilometer). The classical model states that, above a critical density $k_{c}$ (approximately $50 \mathrm{veh} / \mathrm{km}$ in Fig. 2), the traffic state begins to enter in a congestion phase, where vehicles are closer to each other and moving slower, until traffic comes to a full stop, at a jam density $k_{j}$, at about $150 \mathrm{veh} / \mathrm{km}$. The data obtained with SUMO for the conventional traffic are consistent with the continuous line. In Fig. 2, we further represent three upper limits of density values, by vertical dashed lines, for the cases of platoons with 5,8 , and 15 vehicles each, corresponding to the following values of $k: 102,131$, and $169 \mathrm{veh} / \mathrm{km}$, respectively. These are the upper bounds at which a constant spacing platooning system could operate. Once the platoons' formation is already in place, lane capacity is proportional to the speed. The flow is equal to lane capacity, if the vehicle density is maintained. The top of each dashed line is the theoretical flow limit with respect to a given density and speed values. For example, $9443 \mathrm{veh} / \mathrm{h}$ is the lane capacity for the case of a density of $131 \mathrm{veh} / \mathrm{km}$, with a platoon featuring $v=72 \mathrm{~km} / \mathrm{h}, n=8, d=1 \mathrm{~m}$, and $D=30 \mathrm{~m}$ (see Table II). However, for a traffic system to operate with a traffic flow close to $9443 \mathrm{veh} / \mathrm{h}$ (under the aforementioned conditions), the admittance of vehicles should be limited, to ensure that the correspondent maximum density of $131 \mathrm{veh} / \mathrm{km}$ would never be surpassed. Otherwise, congestion would occur, lowering the traffic flow value. Moreover, such traffic system should be based on constant spacing platooning of autonomous vehicles and managed by an ATMS. Based on Table II, we find $n=8$ as the best compromise between traffic flow improvement and platoons' dimension.

\section{Base Model of Vehicle Control}

It becomes clear that one way to effectively improve road capacity consists of the use of vehicle platoons with constant spacing. However, constant spacing platooning requires string stability, which means that the spacing errors do not amplify down the platoon from vehicle to vehicle. To ensure string stability between vehicles in a constant spacing platoon, IVC is required [1], [3].

According to Fig. 3 and considering the desired $i$ th vehicle position defined by

$$
x_{i_{-} \text {des }}=x_{i-1}-L_{i}
$$

where $L_{i}=l_{i-1}+g_{i_{-} \text {des }}$, with $l_{i-1}$ being the length of the preceding vehicle and $\bar{g}_{i \_d e s}$ being the intervehicle desired gap, the spacing error is defined as

$$
\varepsilon_{i}=x_{i}-x_{i-1}+L_{i} .
$$

Using a sliding-mode approach, Rajamani [3] designed a controller with vehicles' desired acceleration expressed as

$$
\begin{aligned}
\ddot{x}_{i \_d e s}= & \left(1-C_{1}\right) \ddot{x}_{i-1}+C_{1} \ddot{x}_{l} \\
& -\left(2 \xi-C_{1}\left(\xi+\sqrt{\xi^{2}-1}\right)\right) \omega_{n} \dot{\varepsilon}_{i} \\
& -\left(\xi+\sqrt{\xi^{2}-1}\right) \omega_{n} C_{1}\left(V_{i}-V_{l}\right)-\omega_{n}^{2} \varepsilon_{i} .
\end{aligned}
$$

The parameters of (6) are described in Table III. With this control method, the spacing error will converge to zero, and the system is string stable, under the conditions $\xi \geq 1$ and $C_{1}<1$ [3]. To each follower in a platoon of vehicles, parameter $C_{1}$ represents the weight factor of the leader's information with respect to the preceding vehicle. When $C_{1}=0$, the following secondorder system is attained:

$$
\ddot{x}_{i \_d e s}=\ddot{x}_{i-1}-2 \xi \omega_{n} \dot{\varepsilon}_{i}-\omega_{n}^{2} \varepsilon_{i}
$$

and only the precedent vehicle's information becomes relevant to each vehicle behavior. From (6), we can conclude that, to determine the desired acceleration of each vehicle, wireless communications are needed to allow each vehicle to receive the speed and acceleration values from both the preceding vehicle and the platoon leader. It is assumed that vehicles have precise 


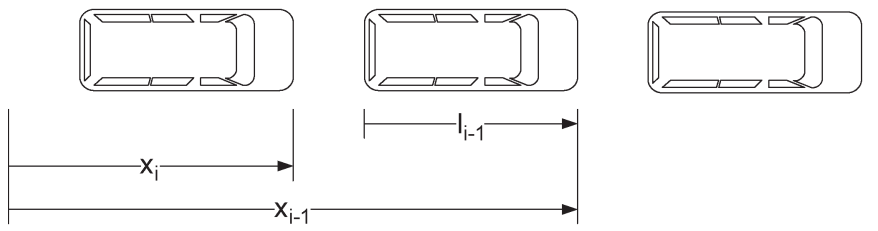

Fig. 3. String of platoon vehicles.

TABLE III

NOMENCLATURE OF MATHEMATICAL FORMULATIONS

\begin{tabular}{ll}
\hline \hline Symbol & Description \\
$\ddot{x}_{i}$ des & desired acceleration of the $i$ th vehicle \\
$\ddot{x}_{i-1}$ & acceleration of the vehicle preceding the $i$ th vehicle \\
$\ddot{x}_{l}$ & acceleration of the vehicle leader of the platoon \\
$V_{i}$ & velocity of the $i$ th vehicle \\
$V_{l}$ & velocity of the vehicle leader of the platoon \\
$C_{1}$ & $\begin{array}{l}\text { control gain used in longitudinal controller } \\
\text { (weight factor of the leader with respect to the }\end{array}$ \\
& preceding vehicle, with values $0 \leq C_{1}<1$ ) \\
$\xi$ & damping ratio (equal to 1 for critical damping) \\
$\omega_{n}$ & bandwidth of the controller
\end{tabular}

self-position information in real time, whether obtained from Global Positioning System (GPS)/differential GPS, or from other sensing means. Moreover, the distance from each vehicle to its precedent is assumed to be determined by the use of a lidar or radar installed in each vehicle. Additionally, it is assumed that platoon formation and leader's election are dealt with by the ATMS at a higher level [13], [14]. The control model does not integrate air drag nor the coefficient of friction effects. As such, the focus of this research is on communication delay effects over an identical set of vehicles and strategies to mitigate them.

\section{COMmunications}

We consider IVC supported by wireless communications from the leader to all followers and from each vehicle to its immediate follower and the leader. The communication scheme uses time-division multiple access with token passing [26]. All vehicles transmit their data at each token cycle $t c$, in their respective time slot $t s$. For that purpose, $t c=10 \times t s_{j}, j=$ $1, \ldots, 10$. We also consider $t c=100 \mathrm{~ms}$ and $t s=10 \mathrm{~ms}$ as appropriate reference values.

\section{Information Management Algorithms}

To exchange information between vehicles, allowing an appropriate platoon behavior, five information-updating schemes are proposed.

Table IV presents the parameters of the information-updating schemes. The delay bounds used in these algorithms are much higher than those obtained through simulation, with respect to packet transmission delays. The idea of these algorithms is to operate under an upper bound delay while continuing to ensure a stable platoon behavior. Consequently, lower delay effects over string stability may then be mitigated or even almost canceled out. Additionally, by managing information at an upper level, with anticipatory information, delays per-
TABLE IV

INFORMATION UPDATING SCHEMES' PARAMETERS

\begin{tabular}{lllrr}
\hline $\begin{array}{l}\text { Algo- } \\
\text { rithm }\end{array}$ & $\begin{array}{l}\text { Antici- } \\
\text { pation }\end{array}$ & C $_{\mathbf{1}}$ & $\begin{array}{r}\text { Apparent Delay } \\
\text { (leader, followers) }\end{array}$ & $\begin{array}{r}\text { Updating } \\
\text { Cycle } \mathrm{uc}\end{array}$ \\
\hline I & none & static & $100 \mathrm{~ms}, 100 \mathrm{~ms}$ & $100 \mathrm{~ms}$ \\
II & leader & static & $0 \mathrm{~ms}, 100 \mathrm{~ms}$ & $100 \mathrm{~ms}$ \\
III & leader & dynamic & $0 \mathrm{~ms}, 100 \mathrm{~ms}$ & $100 \mathrm{~ms}$ \\
IV & all & static & $0 \mathrm{~ms}, 0 \mathrm{~ms}$ & $100 \mathrm{~ms}$ \\
V & all & static & $0 \mathrm{~ms}, 0 \mathrm{~ms}$ & $800 \mathrm{~ms}$ \\
\hline
\end{tabular}

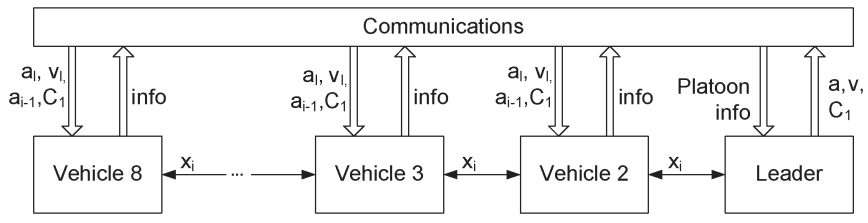

Fig. 4. Platoon's information flow diagram.

ceived by receivers may be canceled out (null apparent delays), as presented in Table IV, and explained throughout the next sections, where the proposed information-updating schemes are described. All of them use the platoon's information flow architecture, which diagram is shown in Fig. 4.

\section{A. Information-Updating Scheme I}

This first scheme is presented as a work base, to which the other proposed schemes II, III, IV, and V will be compared with. Moreover, it also makes possible the study of the influence that $C_{1}$ parameter presents over the platoon stability. The platoon's leader transmits its data to all followers in its time slot $t s_{1}$. Each vehicle $j$, in sequence, transmits its data to the respective follower, in each $t s_{j}$, in sequence. After all vehicles have obtained the data, they proceed to the computation of the next acceleration they must apply through their actuators. However, all vehicles are synchronized and simultaneously update their operation parameters at the end of each information-updating cycle $u c$ to improve platoon stability [26]. We will designate that instant by vehicle actuation moment vam. We adopt an updating cycle $u c=100 \mathrm{~ms}$. With $n$ being the number of vehicles per platoon, with $1 \leq n \leq 8$, and $t s=10 \mathrm{~ms}$, condition $u c \geq n \times t s$ is verified.

Fig. 5 shows a temporal diagram of the packet data exchange between the platoon vehicles in two consecutive 100-ms time frames. Different colors represent different data content. First, the leader broadcasts its present data to all followers. Each of them, in turn and in their respective time slot, transmits the acceleration computed in the previous 100-ms time frame. At the end of the frame, all vehicles compute their next acceleration, based on the measured distance to the respective precedent vehicle, and the data received from that vehicle and the leader. At the same time, the leader computes the acceleration value to apply in the next frame. Then, all vehicles simultaneously perform the actuation of the obtained data in each vehicle. The information of the leader and also of all vehicles to each of their followers is $100 \mathrm{~ms}$ old.

Table $\mathrm{V}$ defines the parameters used in the following algorithms: 

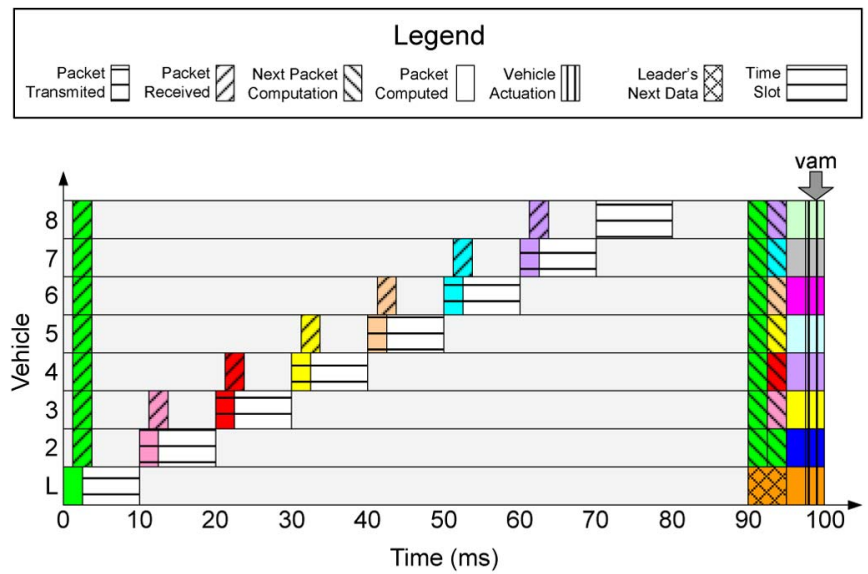

(a)

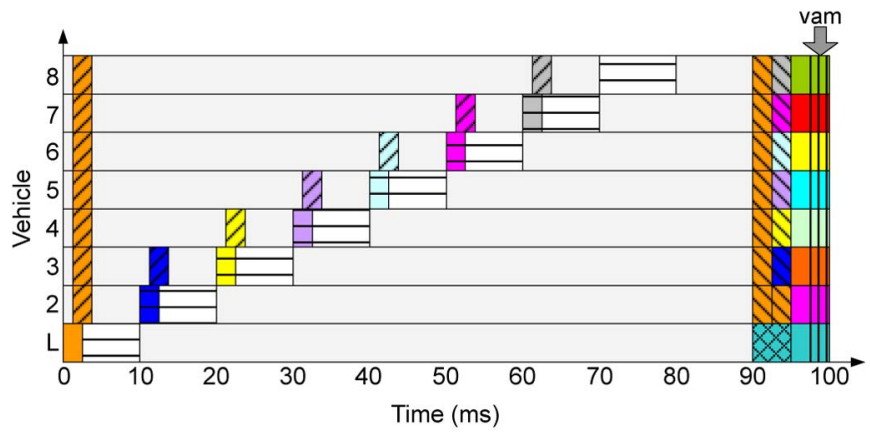

(b)

Fig. 5. Algorithm I: Without anticipatory information. (a) First 100-ms time frame. (b) Second 100-ms time frame.

Algorithm 1 Leader in Information-Updating Scheme I

for all $u c_{i}$ do

At $t s_{1}$, the leader broadcasts its current acceleration $a_{c}$ and speed $v_{c}$.

At $t s_{10}$, the leader gets from ATMS or computes its next acceleration $a_{n}$.

At $v a m$, the leader actuates with $a_{n}$. end for

Algorithm 2 Follower in Information-Updating Scheme I

for all $u c_{i}$ do

At $t s_{1}$, vehicle $v_{j}$ receives the acceleration and speed from the leader.

At $t s_{j-1}$, vehicle $v_{j}$ receives the acceleration from its precedent vehicle.

At $t s_{j}$, vehicle $v_{j}$ transmits its current acceleration $a_{c}$ to its follower.

At $t s_{10}$, vehicle $v_{j}$ compute its next acceleration $a_{n}$ under the control law of (6)

At vam, vehicle $j$ actuates with $a_{n}$ end for
TABLE V

ALGORITHM PARAMETERS

\begin{tabular}{ll}
\hline \hline Parameter & Definition \\
\hline$u c_{i}$ & updating cycle $i$ \\
$t s_{j}$ & time slot $j$ \\
$a_{c}$ & current acceleration \\
$a_{n}$ & next acceleration \\
$v_{c}$ & current speed \\
$v_{p}$ & predicted speed \\
$v a m$ & vehicle actuation moment \\
\hline
\end{tabular}

Algorithm 1 presents the leader's behavior in this information-updating scheme, whereas Algorithm 2 presents the behavior of a follower, both represented in pseudocode.

\section{B. Information-Updating Scheme II}

In this information-updating scheme, the platoon's leader announces the plan for the next frame at each $t c$, in its $t s_{1}$ but waits before actuating with the computed values to allow the followers to be able to update their data before their own actuation. This waiting step is a key feature to improve platoon stability and may be interpreted as an "anticipatory information" that all vehicles receive from the leader to allow them timely and simultaneous actuation, at the vehicle actuation moment vam. The information that each follower emits to the subsequent vehicle is $100 \mathrm{~ms}$ old, as in Scheme I. Each updating cycle is $u c=t c=100 \mathrm{~ms}$.

Fig. 6 shows a temporal diagram of the packet data exchange between the platoon vehicles in two consecutive 100-ms time frames. First, the leader broadcasts the data to all their followers. However, differently from the previous scheme, the information broadcasted by the leader has not yet been applied to the vehicle. Instead, the leader waits until all the remaining vehicles receive and transmit their data. Each vehicle, in turn and in the respective time slot, transmits its actual acceleration (which was computed in the previous 100-ms time frame, considering the leader's anticipatory information). With this updating scheme, it is possible, at the cost of an increase of $100 \mathrm{~ms}$ in the reaction time of the leader, to considerably improve the platoon stability, in the direct proportion of the leader's information weight, as represented by parameter $C_{1}$ in (6).

Algorithm 3 Leader in Information-Updating Scheme II

for all $u c_{i}$ do

At $t s_{1}$, leader computes its predicted speed $v_{p}$ at vam, based on its current acceleration $a_{c}$

At $t s_{1}$, leader broadcasts its next acceleration $a_{n}$ and predicted speed $v_{p}$

At $t s_{10}$, leader gets from ATMS or compute the next acceleration to use at the next updating cycle $u c_{i+1}$

At vam, leader actuates with $a_{n}$

end for 

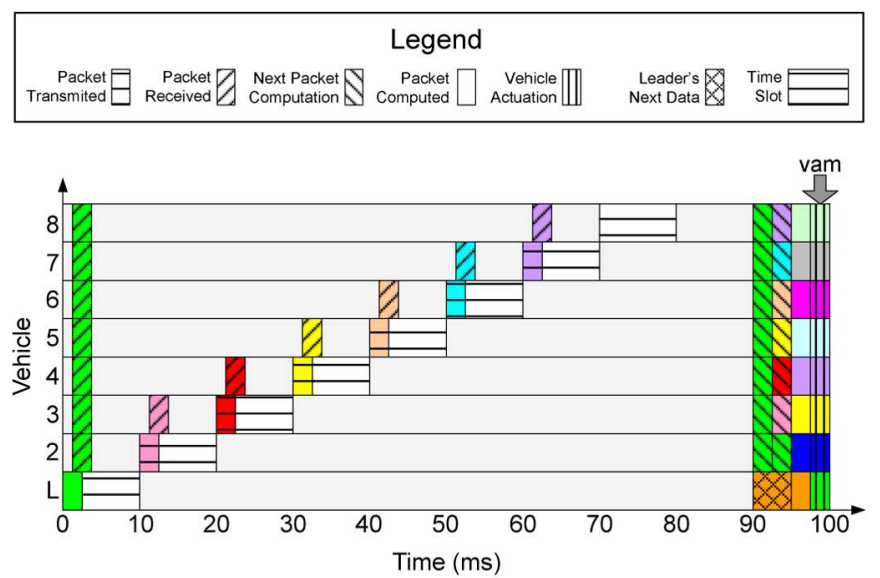

(a)

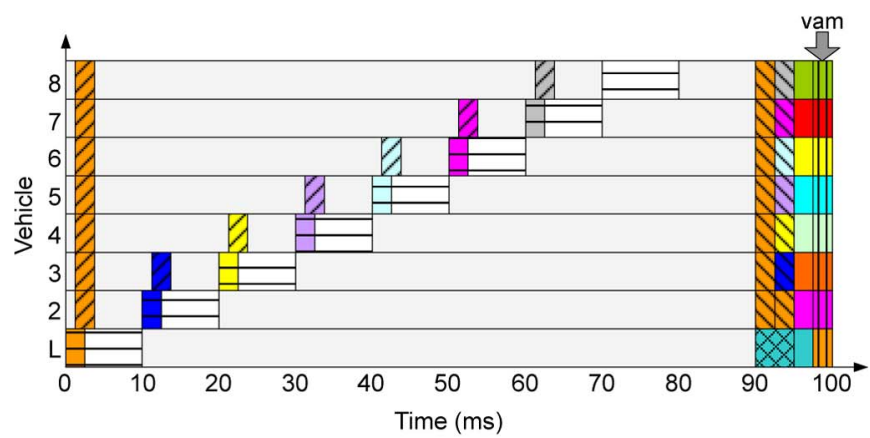

(b)

Fig. 6. Algorithm II: With leader's anticipatory information, using the vehicle actuation moment vam. (a) First 100-ms time frame. (b) Second 100-ms time frame.

Algorithm 3 presents the leader's behavior in this information-updating scheme, whereas the behavior of a follower is the same as in Scheme I (see Algorithm 2). The main differences from Algorithm 1 are presented in italic text.

\section{Information-Updating Scheme III}

All vehicles in the platoon receive information from the leader at all $u c$, as in Scheme II. Parameter $C_{1}$ in (6), which represents the weight of the leader's data, is dynamically changed to cope with some potential dangerous conditions (e.g., in the presence of a unexpected strong deceleration of the leader). In such cases, the leader warns its followers to increase their $C_{1}$. We introduce here a new parameter $C_{2}$ that will serve as a base value, whereas the $C_{1}$ parameter will dynamically vary, which we will designate by $C_{1_{\mathrm{dyn}}}$, where $C_{1}=C_{1_{\mathrm{dyn}}}$, with $C_{2} \leq$ $C_{1_{\mathrm{dyn}}}<1$. This parameter is used when strong accelerations (both positive or negative) are determined by the leader. In these cases, $C_{1_{\text {dyn }}}$ immediately assume a value close to 1 (without reaching it, since string stability requires that $C_{1}<1$ [3]), for a very short period of time, after which, the value rapidly decays to the $C_{2}$ base value again. Nevertheless, all vehicles use the same $C_{1}$ value in each $u c$. With this algorithm, very conservative $C_{2}$ values may be maintained, whereas a very stable platoon behavior is achieved. As we will see in the results (Section VII-C), the behavior of the platoon is even better than the obtained behavior with a fixed $C_{1}=0.9$.

\section{Information-Updating Scheme IV}

This information-updating scheme extends the key feature of Scheme II (anticipatory information) from the leader to all vehicles. The platoon leader announces the plan for the next frame at each $t c$, in its $t s_{1}$. Each follower, in sequence, determines its own plan for the next $t c$ and transmits it to their respective follower, in their own time slot. As such, at each $t c$, both the leader and each of the followers, in sequence, transmit their data. Each vehicle $j$ transmits in its time slot $t s_{j}$ to allow all vehicles to transmit without packet collisions. It is important to note that, although both the leader and all the remaining platoon vehicles transmit their next acceleration value, they all hold their actuation, until all vehicles receive their information. Only then, all vehicles simultaneously update their operation parameters, at the vehicle actuation moment vam, in the end of each information-updating cycle $u c$. Each updating cycle $u c=100 \mathrm{~ms}$. Since this updating scheme transmits data to use on the next $u c$, the platoon stability significantly improves in comparison with the other schemes. The timings at which this algorithm operates are appropriate to demanding dynamic operations, where vehicles are required to be more responsive and adaptive. However, it is very demanding with respect to both communication and timely computation of vehicle parameters.

Fig. 7 shows a temporal diagram of the packet data exchange between the platoon vehicles in a 100-ms time frame. This scheme consists of an evolution of Scheme II. In this case, all vehicles in the platoon announce the acceleration value before the moment at which it will be used to act on the vehicle. For that purpose, a tight synchronization and very short timings must be ensured. Note in Fig. 7(a) that each vehicle has to compute its next acceleration value within the time it received the data of its precedent vehicle and the beginning of its own time slot, where it must be able to transmit the computed value to its own follower. In Fig. 7, we admit that less than one third of the time slot is used to receive the precedent vehicle's data, which leaves enough remaining time to perform the needed computations.

Algorithm 4 Follower in Information-Updating Scheme IV

for all $u c_{i}$ do

At $t s_{1}$, vehicle $j$ receives the acceleration and speed from the leader.

At $t s_{j-1}$, vehicle $j$ receives the acceleration from its precedent vehicle.

At $t s_{j-1}$, vehicle $j$ compute its predicted speed $v_{p}$ at $v a m$, based on its current acceleration $a_{c}$.

At $t s_{j-1}$, vehicle $j$ compute its next acceleration $a_{n}$ at vam, under the control law of (6).

At $t s_{j}$, vehicle $j$ transmits its next acceleration $a_{n}$ to its follower.

At $v a m$, vehicle $j$ actuates with $a_{n}$.

end for 

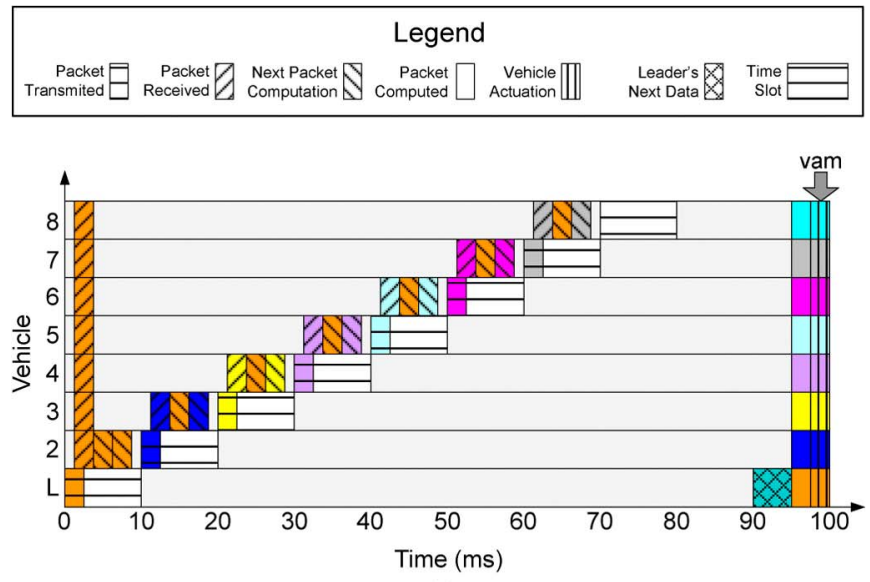

(a)

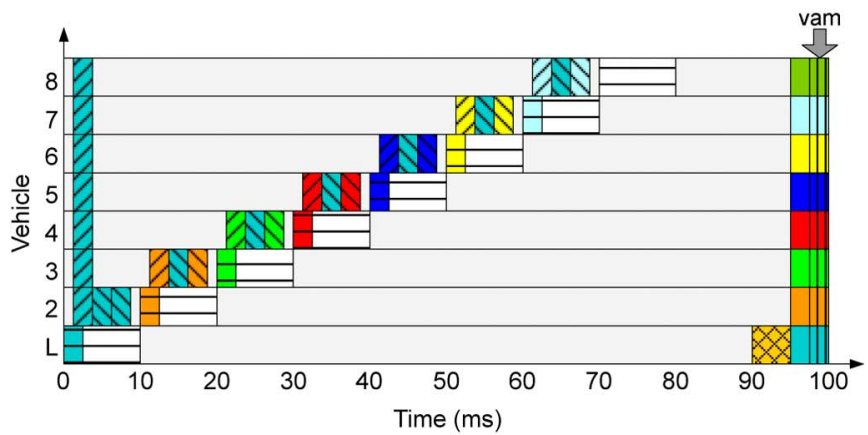

(b)

Fig. 7. Algorithm IV: With all vehicles' anticipatory information, using vehicle actuation moment vam. (a) First 100-ms time frame. (b) Second 100 -ms time frame.

Algorithm 4 presents the follower's behavior in this information-updating scheme, whereas the behavior of a leader is the same as in Scheme II (see Algorithm 3).

\section{E. Information-Updating Scheme $V$}

At the first $t c$, the leader transmits its data to the followers. At each subsequent $t c$, each of the followers, in sequence, transmits their data to the vehicle behind them. As on Scheme IV, the vehicles hold their actuation, until all vehicles receive their information. Only then, all vehicles simultaneously update their operation parameters, at the vehicle actuation moment $v a m$, in the end of each information-updating cycle $u c$. With an $n$-vehicle platoon, each $u c$ should attain the condition $u c \geq$ $n \times t c$. For an eight-vehicle platoon, with $t c=100 \mathrm{~ms}, u c \geq$ $800 \mathrm{~ms}$. This updating scheme is proposed for a stable and foreseeable traffic state. Its major advantage consists of the significant low communication channel load it presents.

\section{RESUlts}

To assess the appropriate operation of the proposed models, several simulations were carried out in Matlab/Simulink [43]. All models include an eight-vehicle platoon of IVC-enabled autonomous vehicles, aiming to maintain a 1-m distance to their precedents. The operational part of the model is implemented in Simulink. Fig. 4 shows the platoon's information flow diagram. The applied acceleration profile and the resultant velocity are

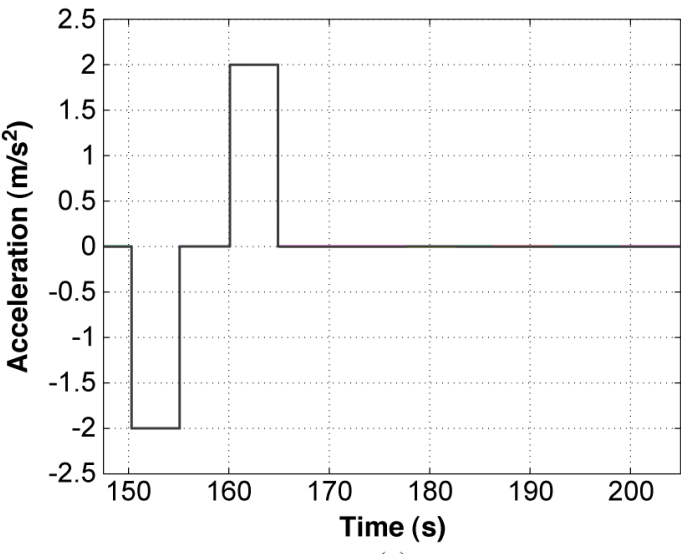

(a)

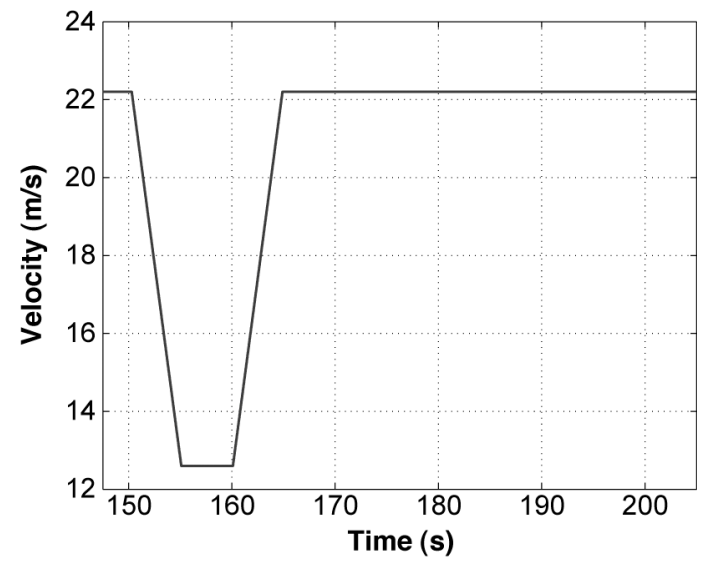

(b)

Fig. 8. Leader's actuation. (a) Acceleration pattern. (b) Velocity pattern.

TABLE VI

Parameters of the Simulation Model

\begin{tabular}{llr}
\hline \hline Parameter & Symbol & Value \\
\hline Time step & $T_{s}$ & $1 \mathrm{~ms}$ \\
Bandwidth of the controller & $\omega_{n}$ & 0.2 \\
Damping ratio & $\xi$ & 1 \\
Leader's weight (fixed) & $C_{1}$ & $0 \leq C_{1}<1$ \\
Leader's weight base value & $C_{2}$ & $0 \leq C_{2}<1$ \\
Leader's weight (dynamic) & $C_{1_{d y n}}$ & $C_{2} \leq C_{1_{d y n}}<1$ \\
Maximum acceleration & $a_{\text {max }}$ & $3 \mathrm{~ms}^{-2}$ \\
Leader's acceleration & $a_{\text {upp }}$ & $2 \mathrm{~ms}^{-2}$ \\
Leader's deceleration & $a_{l o w}$ & $-2 \mathrm{~ms}^{-2}$ \\
Maximum deceleration & $a_{\min }$ & $-4 \mathrm{~ms}^{-2}$ \\
Communication time slot & $t s$ & $10 \mathrm{~ms}$ \\
Communication token cycle & $t c$ & $100 \mathrm{~ms}$ \\
Information updating cycle & $u c$ & $100 \mathrm{~ms}, 800 \mathrm{~ms}$
\end{tabular}

shown in Fig. 8. Table VI presents the simulation parameters used in the Matlab/Simulink simulations.

In this section, we present the results obtained from simulations performed with Matlab/Simulink for the five informationupdating schemes previously proposed.

\section{A. Information-Updating Scheme I}

The first simulation corresponds to Scheme I (Section VI-A). It is assumed that all vehicles operate with transmitted information $100 \mathrm{~ms}$ old. As such, their response will present a delay 
with respect to each precedent vehicle. In Fig. 9(a), the increase in the absolute values of the followers' acceleration with respect to the leader is presented. Fig. 9(b) shows a detailed region of Fig. 9(a), where we can observe the discrete values of the acceleration, with a 100-ms sample period. Fig. 9(c) shows the resultant velocity of each vehicle, with all of them approaching their precedent vehicle and subsequently falling behind it, as Fig. 9(d) clearly shows. All vehicles reach to a point (at about $155 \mathrm{~s}$ ) where they are only $60 \mathrm{~cm}$ apart from the respective precedent vehicle. In this first simulation, we used $C_{1}=0$, which means that the leader's information has no effect on the behavior of all the platoon followers, except on the second vehicle, since this is the leader's immediate follower.

A second simulation was performed using $C_{1}=0.999$. Fig. 10 shows the acceleration, velocity, and spacing error of this case. Note that the second vehicle is noticeably affected by the 100-ms communication delay, presenting a worse response than that of the previous case. Equation (6) may enlighten this issue. When $C_{1}=0$, the computation of the next acceleration value does not consider the difference of the velocity values between the vehicle and the leader. However, as $C_{1}$ increases, that difference is used. Unfortunately, the second vehicle is using 100-ms-old information, from both the leader's acceleration and the velocity values. As such, the result is worse when compared with the case of $C_{1}=0$ since the spacing error in the later case was only caused by 100-ms-old acceleration value from the precedent vehicle (which is the leader in this case). The response is also more abrupt than before. Since the remaining vehicles receive the same information from the leader at the same time, the spacing error toward their precedent vehicles is softened. (It is worth remembering that they are operating with $C_{1}=0.999$.)

\section{B. Information-Updating Scheme II}

This scheme differs from the previous scheme by a feature that is of the most importance to the stability of the platoon: the transmission of leader's anticipatory information. As explained in Section VI-B, this is due to the leader's waiting step, until the vehicle actuation moment vam is reached, allowing its followers to timely use the transmitted information in their own next acceleration computation. The simulation of this scheme presents several relevant results: as we can observe from Fig. 11(a), independently of the value of $C_{1}$, the second vehicle mimics the behavior of the leader, as it is also its precedent. The third vehicle response is shown in Fig. 11(b). The response is identical of that of Scheme I when $C_{1}=0$, as expected. However, as $C_{1}$ increases, the vehicle's response is considerably improved. As $C_{1}$ approaches 1, the tracking error approaches zero. The same behavior is presented by all the remaining vehicles [the eighth vehicle's response is shown in Fig. 11(c). These results show that the leader's anticipatory information has a major impact on the platoon stability, and apart from the second vehicle, its effect is proportional to $C_{1}$. Ideally, platooning with this operating scheme and a very high $C_{1}$ value would assure a very stable platoon behavior. However, it would be also problematic in the long run since vehicles would be considering the leader's data almost exclusively,

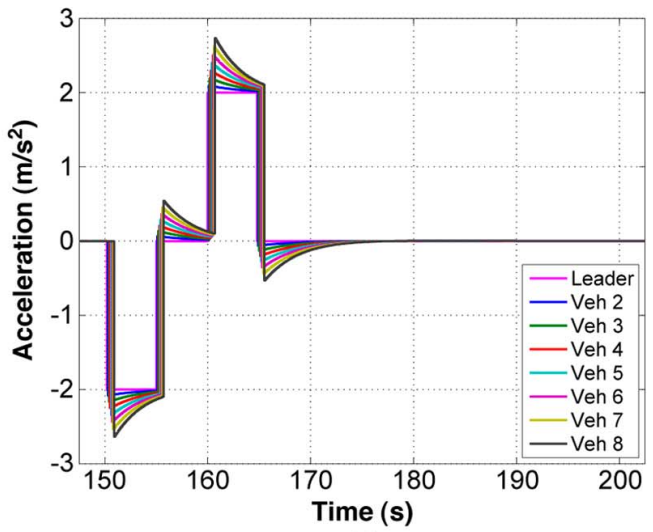

(a)

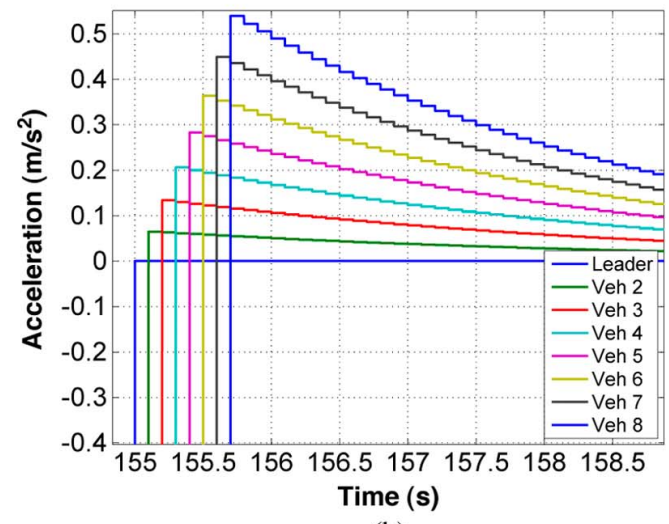

(b)

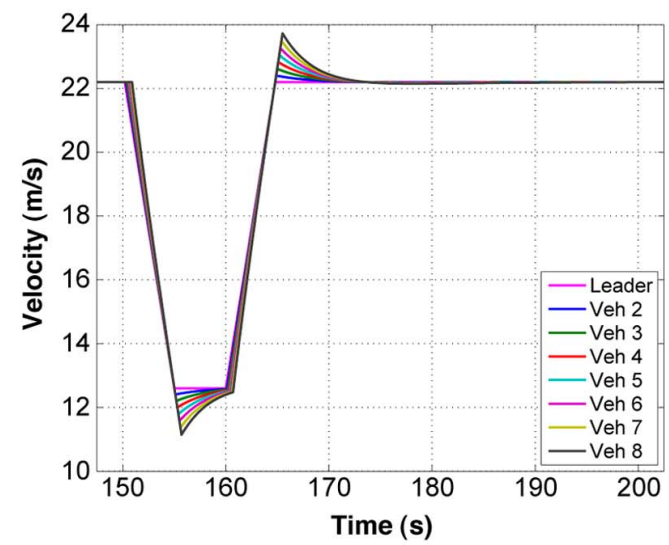

(c)

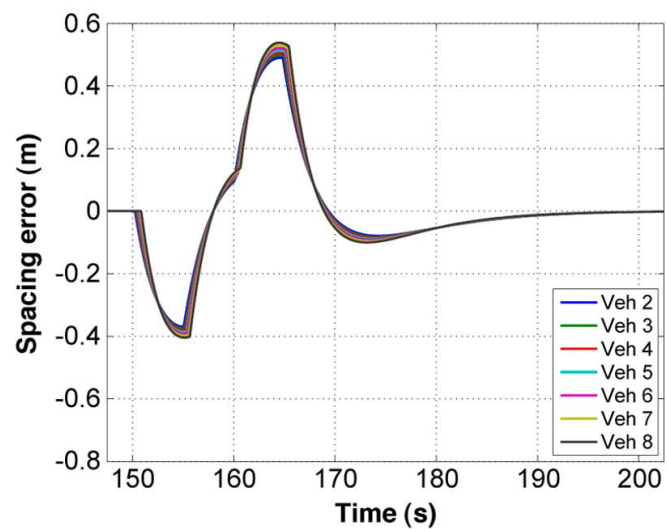

(d)

Fig. 9. Algorithm I with $C_{1}=0$. (a) Acceleration of the platoon. (b) Acceleration of the platoon (zoomed). (c) Velocity of the platoon. (d) Spacing error of the platoon. 


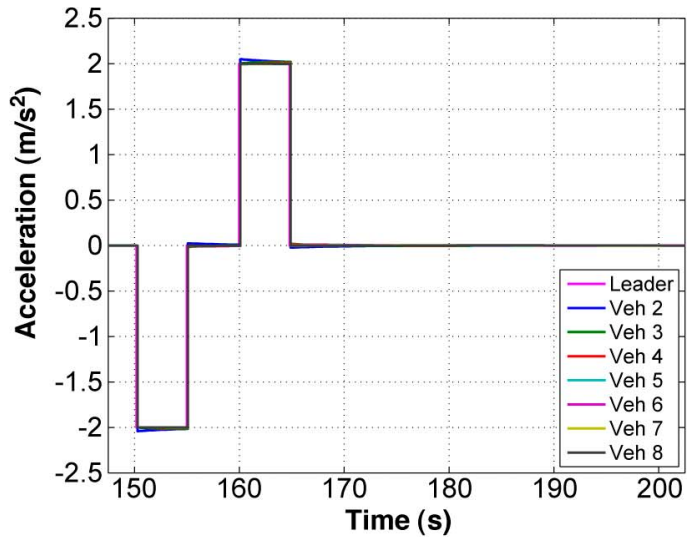

(a)

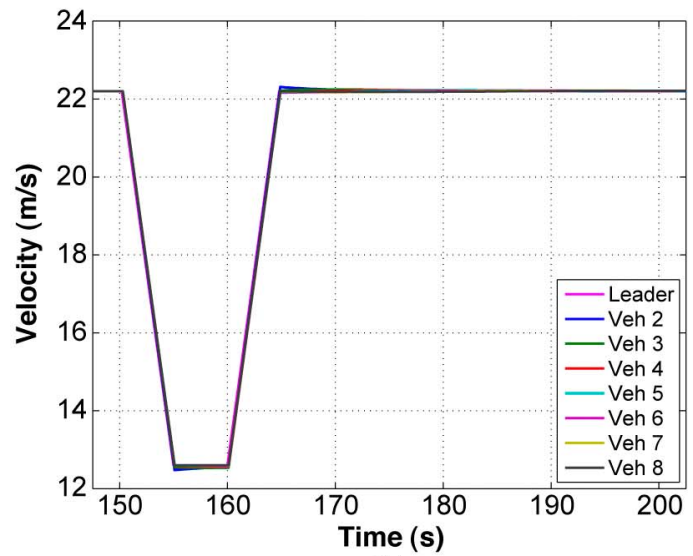

(b)

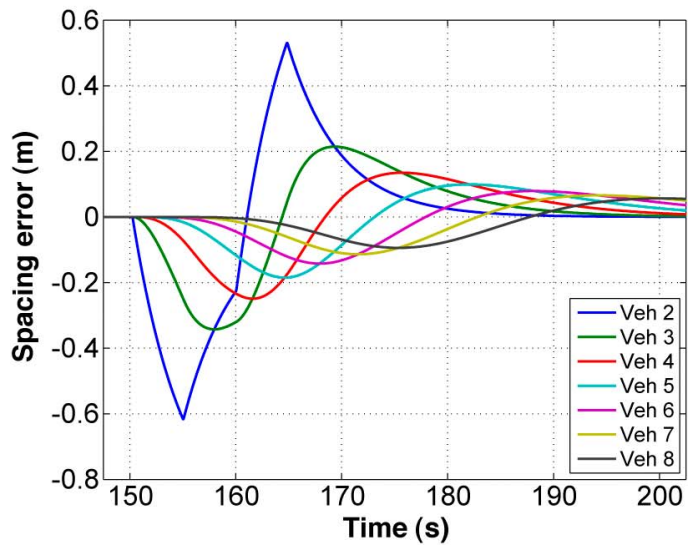

(c)

Fig. 10. Algorithm I with $C_{1}=0.999$. (a) Acceleration of the platoon. (b) Velocity of the platoon. (c) Spacing error of the platoon.

which is neither secure nor reliable. As such, a scheme that could entangle a balance between stability and safety would be preferable. The platoon operating with a fixed $C_{1}$ value between 0.5 and 0.7 has shown to be an acceptable compromise. However, it is not adaptive.

\section{Information-Updating Scheme III}

In the simulation of this updating scheme, we analyze the adaptive value for the leader's information weight $C_{1_{\mathrm{dyn}}}$, discussing in detail the effects of this dynamic parameter on the platoon stability. Table VII presents the spacing errors of

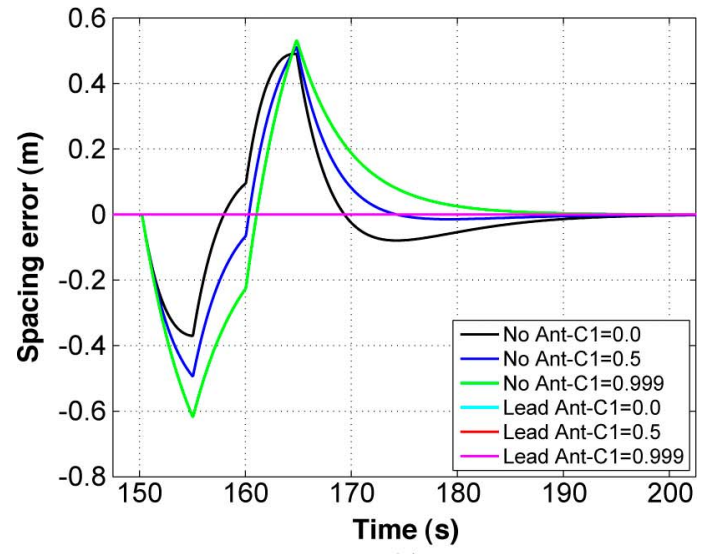

(a)

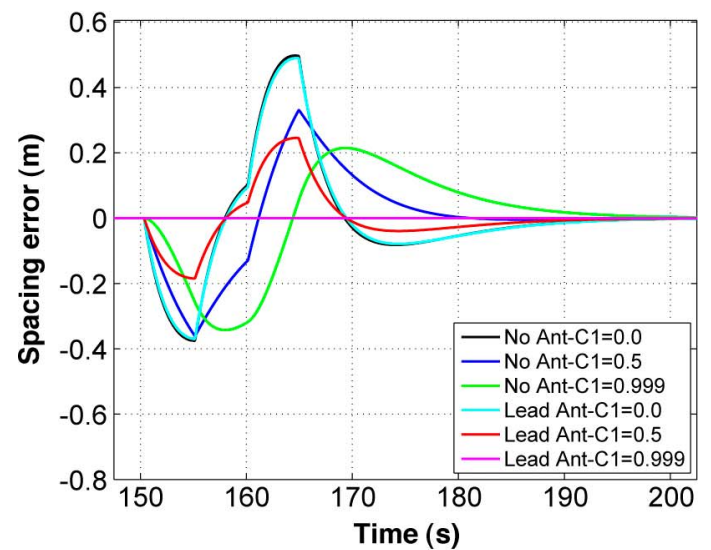

(b)

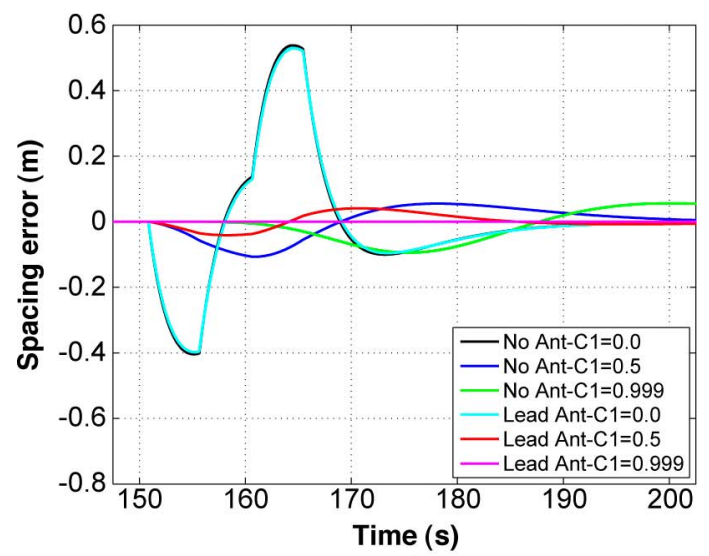

(c)

Fig. 11. Algorithm II: Leader's anticipation. (a) Vehicle 2. (b) Vehicle 3. (c) Vehicle 8

algorithms I and II, when performed with several different $C_{1}$ parameter values. The lines in bold represent the spacing error values when the vehicles approach their precedent, with respect to the 1-m objective distance (negative error values), whereas the other lines represent the spacing error when the vehicles have fallen behind its 1-m objective (positive error values). We can observe that the $C_{1}$ value is crucial to platoon stable behavior.

When operating with $C_{1}=0.5$, the spacing error data of the vehicles in positions 4,5 , and 6 seem to be contradictory. Therefore, a more detailed analysis is required. In fact, these vehicles seem to present no behavior improvement from the 
TABLE VII

INFLUENCE OF $C_{1}$ ON THE SPACING ERROR

\begin{tabular}{|c|c|c|c|c|c|c|c|c|c|}
\hline \multirow{2}{*}{ Veh. } & \multicolumn{3}{|c|}{$\mathrm{C}_{1}=\mathbf{0}$} & \multicolumn{3}{|c|}{$\mathrm{C}_{1}=0.5$} & \multicolumn{3}{|c|}{$\mathrm{C}_{1}=0.9$} \\
\hline & NA & LA & $\%$ & NA & LA & $\%$ & NA & LA & $\%$ \\
\hline \multirow{2}{*}{2} & -0.370 & 0.000 & 100 & -0.493 & 0.000 & 100 & -0.592 & 0.000 & 100 \\
\hline & 0.490 & 0.000 & 100 & 0.509 & 0.000 & 100 & 0.526 & 0.000 & 100 \\
\hline \multirow{2}{*}{3} & -0.375 & -0.370 & 1 & -0.360 & -0.185 & 49 & $-\mathbf{0 . 3 2 7}$ & $-\mathbf{- 0 . 0 3 7}$ & 89 \\
\hline & 0.497 & 0.490 & 1 & 0.330 & 0.245 & 26 & 0.213 & 0.049 & 77 \\
\hline \multirow{2}{*}{4} & -0.381 & -0.375 & 2 & -0.257 & -0.139 & 46 & -0.238 & -0.020 & 91 \\
\hline & 0.505 & 0.497 & 2 & 0.190 & 0.180 & 5 & 0.131 & 0.026 & 80 \\
\hline \multirow{2}{*}{5} & -0.386 & -0.381 & 1 & -0.187 & -0.101 & 46 & -0.177 & -0.012 & 93 \\
\hline & 0.513 & 0.505 & 2 & 0.120 & 0.123 & -3 & 0.095 & 0.015 & 85 \\
\hline \multirow{2}{*}{6} & -0.392 & -0.386 & 2 & -0.151 & -0.073 & 52 & $-\mathbf{- 0 . 1 3 5}$ & $\begin{array}{l}-0.009 \\
\end{array}$ & 94 \\
\hline & 0.521 & 0.513 & 2 & 0.087 & 0.079 & 9 & 0.075 & 0.010 & 87 \\
\hline \multirow{2}{*}{7} & -0.398 & -0.392 & 2 & -0.128 & -0.052 & 59 & -0.108 & -0.006 & 94 \\
\hline & 0.529 & 0.521 & 2 & 0.068 & 0.055 & 19 & 0.062 & 0.007 & 89 \\
\hline \multirow{2}{*}{8} & -0.404 & -0.398 & 1 & -0.107 & -0.041 & 62 & -0.089 & -0.005 & 95 \\
\hline & 0.538 & 0.529 & 2 & 0.055 & 0.041 & 25 & 0.053 & 0.005 & 90 \\
\hline LEGENI & \multicolumn{7}{|r|}{ oation } & b-Impro & \\
\hline
\end{tabular}

leader's anticipatory information with respect to the case where no anticipation exists. In Fig. 12, we can see the behavior of the platoon with $C_{1}=0.5$. Fig. 12(a) shows the spacing error with no anticipatory information, whereas Fig. 12(b) shows the case when anticipation exists. We can observe that, in the later case, since the second vehicle mimics the behavior of the leader, the remaining vehicles are unable to so promptly respond when acceleration occurs. Therefore, the response of the vehicles in positions 4, 5, and 6 is not as good as expected, resulting in similar values of the former case, in the absence of anticipatory information, as can be seen in Fig. 12(c). However, it is important to notice that this only happens when vehicles accelerate. During braking maneuvers, the improvement is always above $45 \%$, which assures a safe operation of the system.

Although higher values of $C_{1}$ prove to be generally beneficial in the presence of leader's anticipatory information, they should not be maintained for a long time. In fact, a high $C_{1}$ value is only required when a considerable difference of the acceleration, in absolute terms, occurs between consecutive $u c$ intervals. When facing strong accelerations and/or decelerations, a prompt platoon response is required to maintain stability. However, as the acceleration stabilizes, $C_{1}$ may then be relaxed to a more conservative value. These are the premises of the present scheme. For this purpose, we introduced the aforementioned parameter $C_{2}$, representing the base value above which the dynamic $C_{1}$, which is represented by $C_{1_{\mathrm{dyn}}}$, will vary, when strong accelerations are determined by the leader. Fig. 13 shows the comparison of the results for equal values of a fixed $C_{1}$ and a base value $C_{2}$ with dynamic $C_{1_{\mathrm{dyn}}}$. We consider only fixed $C_{1}$ values of 0.7 and 0.9 since the difference of results using low fixed $C_{1}$ with respect to equally low $C_{2}$ with dynamic $C_{1_{\text {dyn }}}$ is so high that the resultant graph scale does not allow accurate visualization of the spacing errors corresponding to the $C_{1_{\mathrm{dyn}}}$ values. Fig. 14 shows the spacing error results showing better response of dynamic $C_{1_{\mathrm{dyn}}}$ with a base value $C_{2}$ of zero, when compared with fixed $C_{1}=0.99$. Note that this is one of the most unfavorable case for $C_{1_{\mathrm{dyn}}}$ and an extremely favorable case for the fixed $C_{1}$. Even so, the dynamic scheme presents a considerable stability improvement. Fig. 15 shows both the acceleration patterns of the third vehicle and the values of dynamic $C_{1_{\mathrm{dyn}}}$ with a base value $C_{2}=0$, and a fixed $C_{1}=0.99$.

\section{Information-Updating Scheme IV}

The results of this scheme are extremely favorable. We already observed, in Scheme II, the beneficial results of the leader's anticipatory information over the platoon behavior, even operating with 1-uc-old information from precedent vehicles. However, with Scheme IV, we took a step further with respect to information anticipation. In this scheme, all vehicles announce the acceleration value that they will use on the next updating cycle $u c$ before their followers compute their own predictable values in an ordered chain of events. Then, all the platoon's vehicles simultaneously actuate at the vehicle actuation moment vam, resulting in a very stable behavior. In this updating scheme, $C_{1}$ may present a near zero value, as far as no communication failures occur. This happens because the leader's information is already incorporated into the precedent vehicles' own information, which is anticipatively received. The results of this algorithm, for different $C_{1}$ values, present a spacing error virtually null.

\section{E. Information-Updating Scheme $V$}

This scheme is analogous of the precedent, with one major difference: $u c$ is now of $800 \mathrm{~ms}$, instead of $100 \mathrm{~ms}$, as in the previous case. This means that only one vehicle transmits at each $t c=100 \mathrm{~ms}$. This scheme is intended to be used in the presence of stable and foreseeable traffic conditions and is very benign in what concerns communication network load. Despite a $u c$ value eight times greater than before, the results are very favorable. The results of this algorithm, for different $C_{1}$ values, present a spacing error virtually null, similarly to the previous scheme case.

\section{NETWORK SIMULATIONS}

Some of the information-updating schemes used in previous simulations are very benign where communication network 


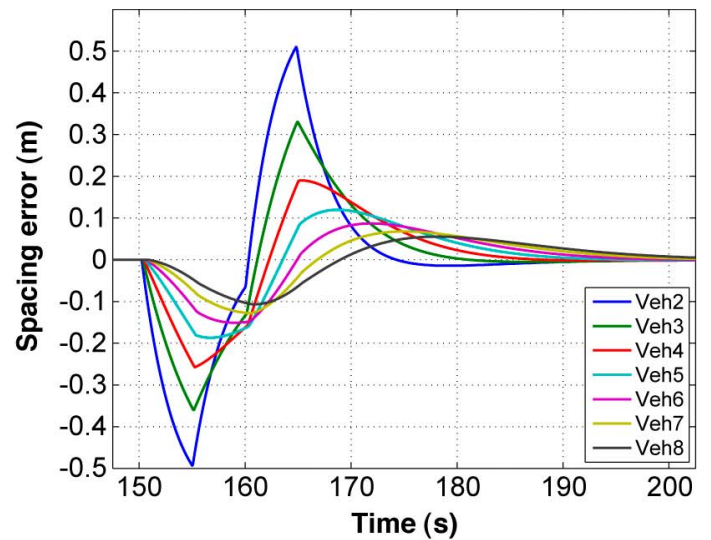

(a)

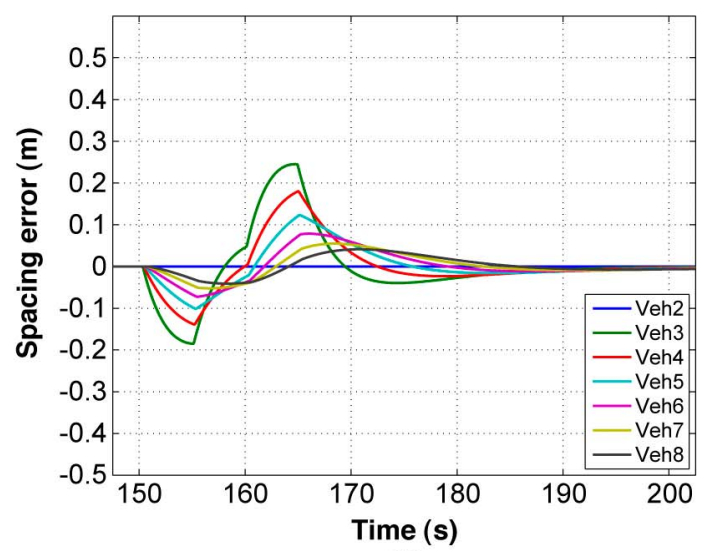

(b)

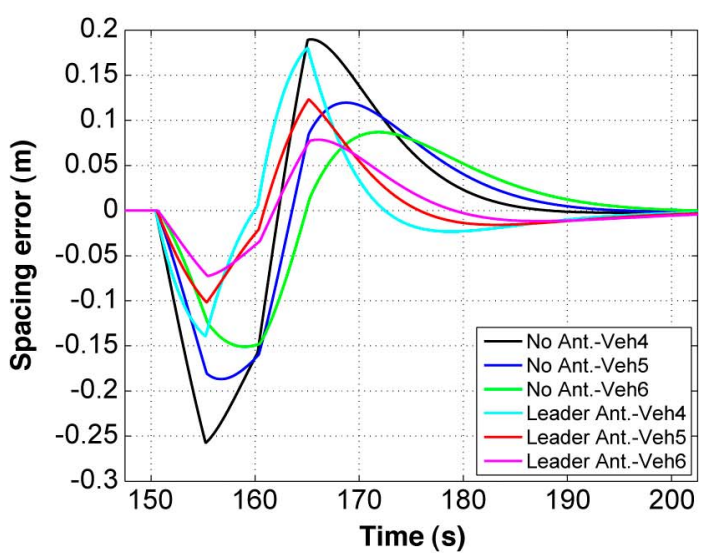

(c)

Fig. 12. Analysis of the influence of $C_{1}=0.5$ on the platoon behavior. (a) No anticipation with $C_{1}=0.5$. (b) Leader's anticipation with $C_{1}=0.5$. (c) Vehicles 4, 5, and 6 comparison, with $C_{1}=0.5$.

load is concerned. However, the current IVC technologies and protocols rise some concerns of whether they can cope with the involved timings of the information-updating schemes, when all vehicles communicate within $100 \mathrm{~ms}$. To assess current IVC operation in such scenarios, we chose to test DSRC since it is conceived to deal with high-mobility patterns, presenting very low latencies and a large-enough range. However, its intrinsic operating characteristics require a more throughout analysis to assess its behavior when a large number of vehicles are involved. For that purpose, we used the NS-3 network simulator.

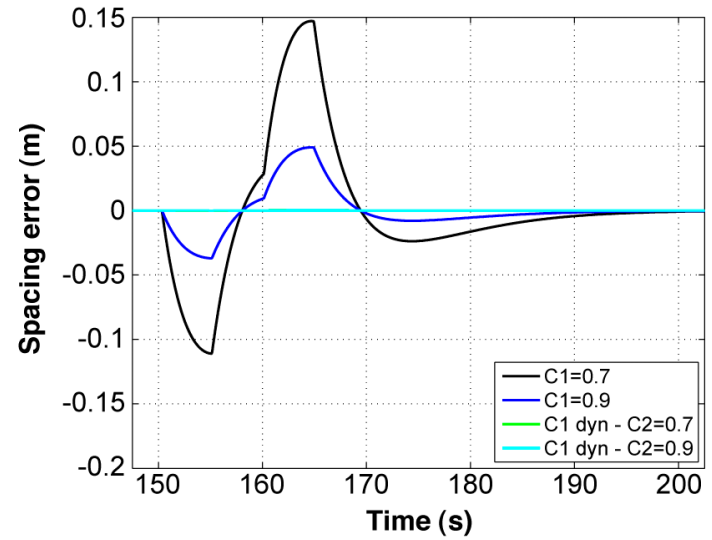

(a)

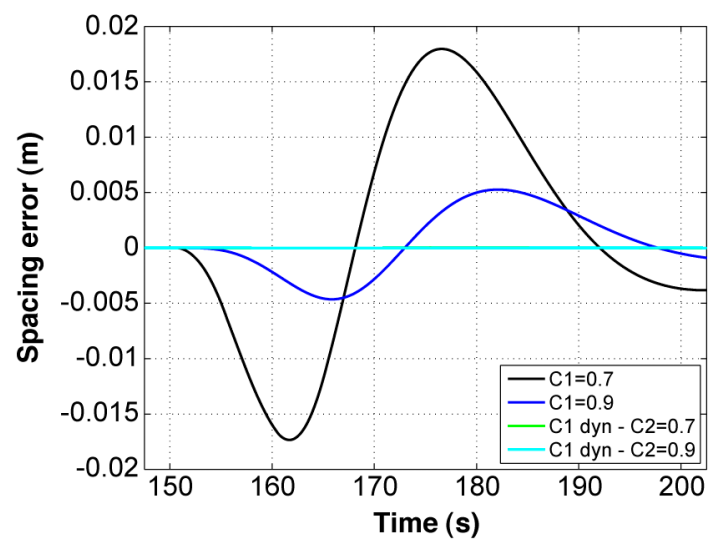

(b)

Fig. 13. Algorithm III: Comparison of a fixed and a dynamic $C_{1}$. (a) Vehicle 3. (b) Vehicle 8.

TABLE VIII

DSRC SYSTEM PARAMETERS

\begin{tabular}{lr}
\hline \hline Parameter & Value \\
\hline Frequency Spectrum & $5.9 \mathrm{GHz}$ \\
Channel Size & $10 \mathrm{MHz}$ \\
Max Range & $\leq 1 \mathrm{Km}$ \\
Data Rate & $3 \mathrm{Mbps}$ to $27 \mathrm{Mbps}$ \\
Latency & $\leq 50 \mathrm{~ms}$ \\
Slot Time ST & $16 \mu \mathrm{s}$ \\
SIFS & $32 \mu \mathrm{s}$ \\
AIFS & $64 \mu \mathrm{s}$ \\
\hline
\end{tabular}

\section{A. DSRC Operating Parameters}

DSRC/Wireless Access in Vehicular Environments are defined in the IEEE 802.11p and 1609.x standards [5]-[7], [44]. The lower layers are defined in IEEE 802.11p, and its MAC protocol uses an improved version of the distributed coordination function, which is named enhanced distributed channel access (EDCA), with quality-of-service (QoS) capabilities. The DSRC system parameters are presented in Table VIII.

\section{B. NS-3 Simulation Setup and Parameters}

A scenario of ten platoons of eight vehicles each was implemented in NS-3. Each platoon is separated by $30 \mathrm{~m}$. Each vehicle is $3 \mathrm{~m}$ long and is separated from the precedent vehicle by $1 \mathrm{~m}$. The antennas are defined at $1.5 \mathrm{~m}$ above the ground. 


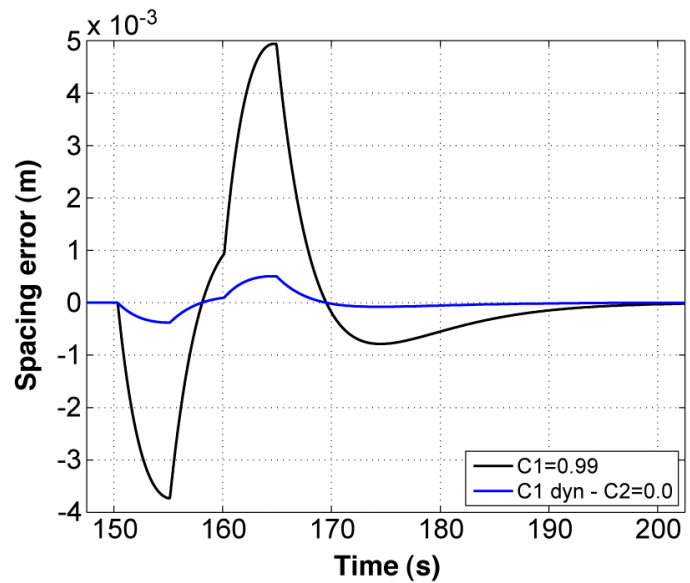

(a)

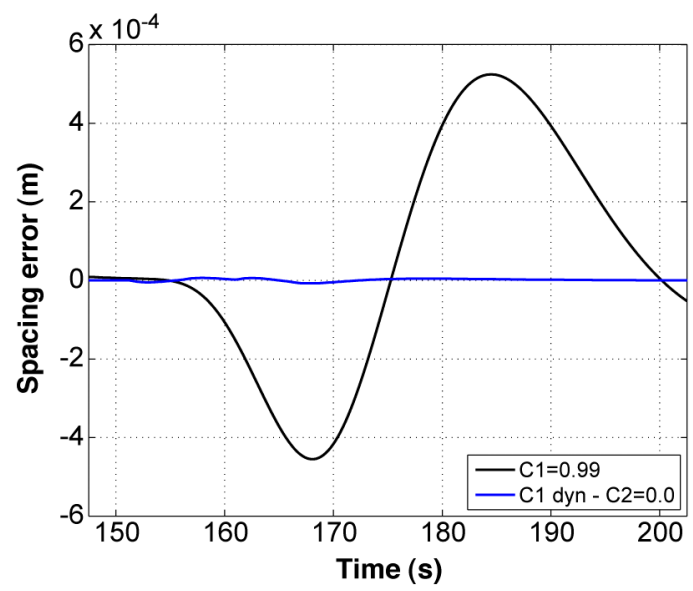

(b)

Fig. 14. Algorithm III: High fixed versus dynamic $C_{1}$ with low $C_{2}$. (a) Vehicle 3. (b) Vehicle 8.

The platoons' leaders broadcast messages to their followers at $3-\mathrm{Mb} / \mathrm{s}$ data rate and in LOS. Each follower communicates to its own follower using unicast at $27-\mathrm{Mb} / \mathrm{s}$ data rate. Each data packet has a 100-B payload, and explicit acknowledgement (ACK) packets have $10 \mathrm{~B}$. The communication is performed through the DSRC control channel $(\mathrm{CCH})$ since $\mathrm{CCH}$ is dedicated to safety and control messages, whereas service channels (SCHs) are reserved for more general-information purposes. The CCH intervals are active for $50 \mathrm{~ms}$ of each $100-\mathrm{ms}$ interval. A 4-ms guard interval (GI) was reserved at the beginning of each $\mathrm{CCH}$ to allow proper synchronization and channel switching of all vehicles [45]. Of the remaining $46 \mathrm{~ms}$, we reserved a time slot of $11 \mathrm{~ms}$ for the leader and 5-ms time slots for each one of the seven followers. Fig. 16 shows the proposed logical division of the $\mathrm{CCH}$, aiming to avoid packet collisions within each platoon. Nevertheless, data packets continue to contend for the transmission media access between each $i$ th vehicle of near platoons.

The main concern that this setup poses is related to proper reception of leaders' broadcasted messages by the vehicles in eighth position at each platoon. In fact, the distance to their respective leaders is $28 \mathrm{~m}$, and the leaders of the platoons behind them are only $33 \mathrm{~m}$ apart (considering antenna-toantenna distances). To assess proper communication flow, a

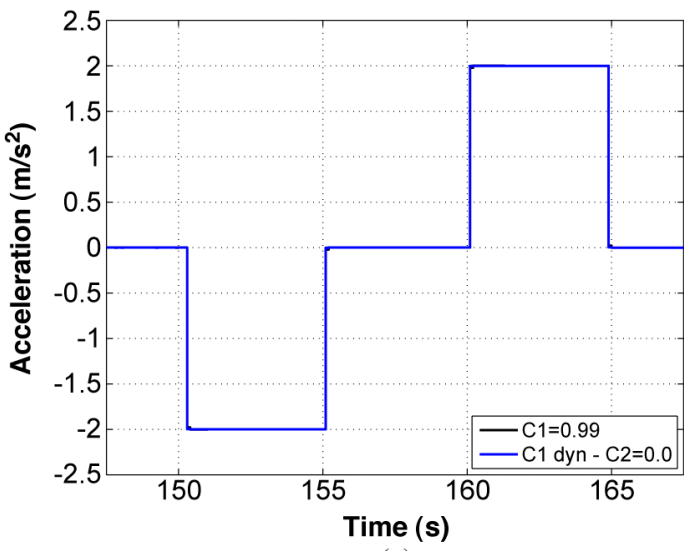

(a)

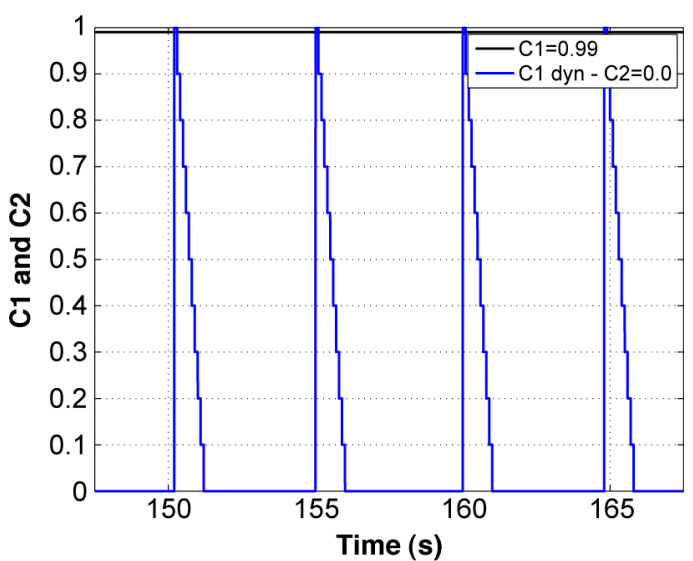

(b)

Fig. 15. Algorithm III: Leader's acceleration and dynamic $C_{1}$. (a) Vehicle 3 : Acceleration. (b) Vehicle 3: Dynamic $C_{1}$ with $C_{2}=0$.

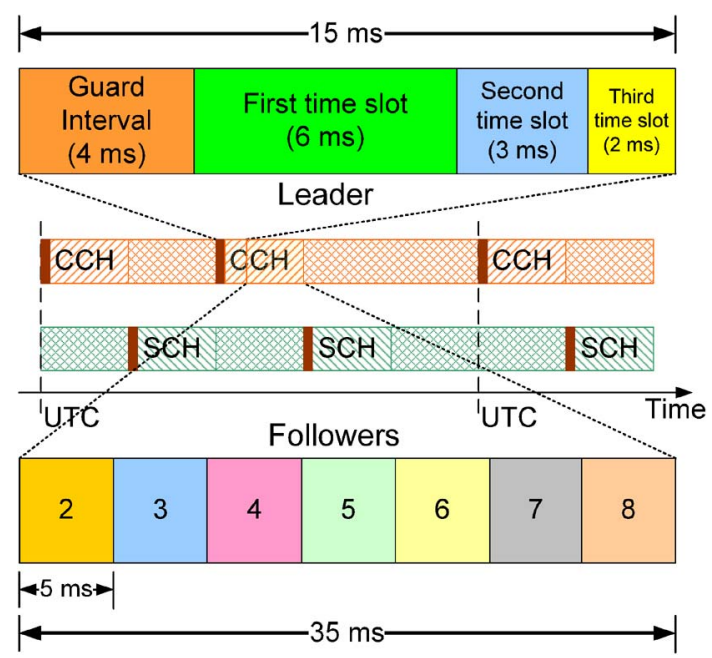

Fig. 16. DSRC CCH leaders' and followers' time slots.

ten-platoon setup of eight vehicles each was implemented. The main simulation parameters are presented in Table IX.

\section{NS-3 Simulation Results}

Several simulations were performed. In the beginning of each one, a warm-up process was conducted to proceed to the fulfillment of the MAC/Internet Protocol address association tables 
TABLE IX

PARAMETERS OF THE NS-3 NETWORK SimUlation

\begin{tabular}{lr}
\hline \hline Parameter & Value \\
\hline Number of nodes & 80 \\
Guard interval & $4 \mathrm{~ms}$ \\
Time slot (leader) & $11 \mathrm{~ms}$ \\
Time slot (follower) & $5 \mathrm{~ms}$ \\
Node spacing (intra-platoon) & $4 \mathrm{~m}$ \\
Node spacing (inter-platoon) & $33 \mathrm{~m}$ \\
Propagation delay model & Constant speed \\
Propagation loss model & Log distance \\
Data rate (leader) & $3 \mathrm{Mbps}$ \\
Data rate (follower) & $27 \mathrm{Mbps}$ \\
$A C$ & $3(\mathrm{VO})$ \\
$A I F S N$ & 2 \\
$C W_{\min }$ & 3 \\
$C W_{\max }$ & 7 \\
\hline
\end{tabular}

(among other actions). This procedure intends to avoid the overhead that such packet exchange would produce during the first superframe of data transmission. DSRC protocol defines that each $\mathrm{CCH}$ start time is synchronized with the coordinated universal time (UTC). Additionally, we divided each $\mathrm{CCH}$ in time slots to avoid intraplatoon collisions. These two features could lead to an interplatoon transmission synchronism, which might increase first packet collisions in the beginning of each time slot, even when using the arbitration interframe space number (AIFSN) and contention window (CW) mechanisms, of the IEEE 802.11e EDCA QoS enhancements [46]. In fact, when a node detects that the media is busy, a new CW is computed, and the corresponding backoff time is applied, i.e.,

$$
C W_{\text {new }}:=\min \left\{2 \times\left(C W_{\text {old }}+1\right)-1, C W_{\text {max }}\right\} .
$$

However, at the beginning of each time slot, only the $A I F S[A C]$ interval is used, with

$$
A I F S[A C]=A I F S N[A C] \times S T+S I F S
$$

with the access category $A C=3\left(A C_{-} V O\right)$, the arbitration interframe space $A I F S[A C]=64 \mu \mathrm{s}, \operatorname{AIFSN}[A C]=2$, DSRC slot time $S T=16 \mu \mathrm{s}$, and the short interframe space $S I F S=32 \mu \mathrm{s}$. Since all platoons' vehicles use this same value, collisions are prone to occur. To minimize the probability of these early collisions, an additional application-level random backoff between $C W_{\min }$ and $C W_{\max }$ was inserted.

The presented data of Figs. 17 and 18 were obtained from statistically noncorrelated simulations of 100 frames of $100 \mathrm{~ms}$ each.

Fig. 17 shows the aggregate data of average, minimum, and maximum values of reception delays from precedent vehicles, per platoon, using unicast. Higher average delay values are presented by the middle platoons. These results are in concordance with what would be expected since these platoons receive transmissions from both the platoons upfront and the platoons behind. (The DSRC range limit may be higher than the length of a ten-platoon chain like this one.) We see that the average values are all under $1 \mathrm{~ms}$, and no failures occurred over the entire simulation. Since the time slots of the followers are $5 \mathrm{~ms}$ long, they have enough remaining time to perform

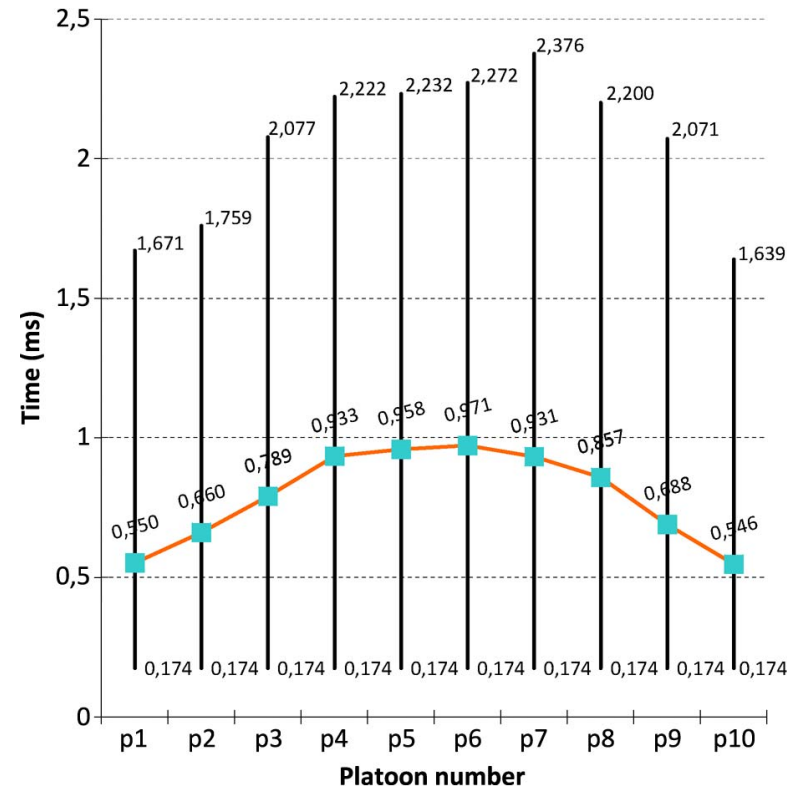

Fig. 17. Platoons' follower reception delays of messages from their precedent vehicles, using unicast.

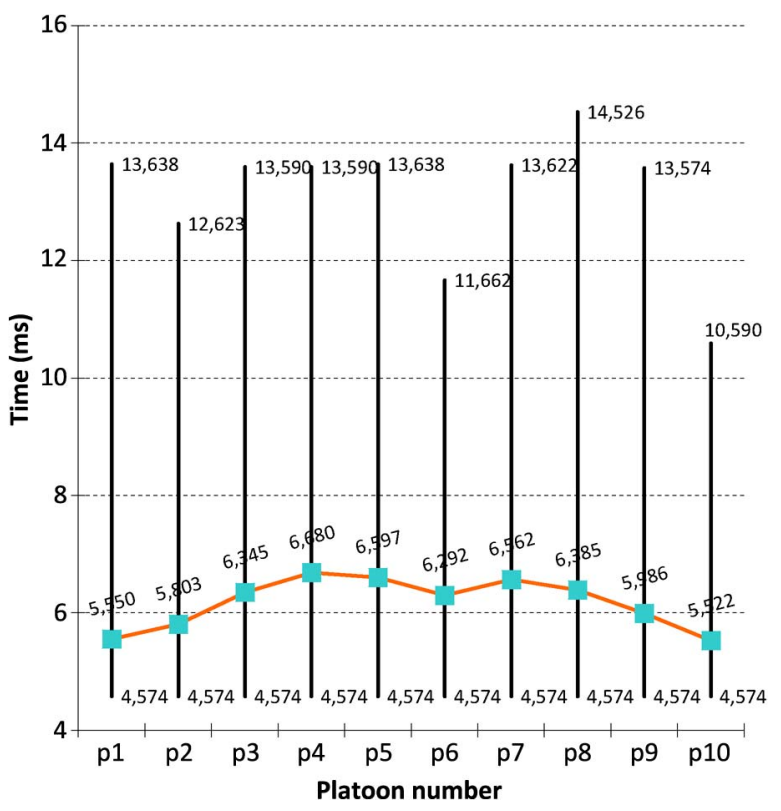

Fig. 18. Platoons' follower reception delays of messages broadcasted by their leaders.

all the required computations, which validates the assumptions made when information-updating Scheme IV was presented and simulated (see Sections VI-D and VII-D, respectively). Even the maximum detected delay of $2.376 \mathrm{~ms}$ is below half the time slot value.

Although unicast communication between followers is reliable, the broadcasted communication between the leaders and their followers is not very resilient. There is no packet delivery guarantee since the ACK mechanism does not exist in broadcasting. Moreover, even if a collision occurs, the broadcast emitter has no means of detecting it since the MAC of IEEE $802.11 \mathrm{p}$ uses carrier sense multiple access with collision avoidance. Therefore, an explicit ACK mechanism had to be 
implemented to ensure proper reception of broadcasted messages from each platoon's leader to all its followers. Although several strategies to improve reliable broadcasting in vehicular environment have been proposed by the research community, this is a problem that has yet to be completely tackled. After several experimental simulations, we decided to implement a selective ACK mechanism. Since all performed simulations revealed that the vehicles in the eighth position had always failed to receive its message when transmissions failure occurred, we used those vehicles as referees to acknowledge successful platoons' leader transmissions. As such, they became the sole followers to emit ACKs upon proper reception of their own leader's messages, in the right time slots. Using this scheme, several subdivisions of the leader's time slots, and two or three transmissions were thoroughly tested. The most favorable results were obtained with the leader's time slot subdivision shown in Fig. 16. Considering the $4 \mathrm{~ms}$ of the GI, the first transmission occurs at $4 \mathrm{~ms}$ from the beginning of each $\mathrm{CCH}$ interval, at the beginning of the 11-ms leader's time slots, and is $6 \mathrm{~ms}$ long; the second one occurs at $10 \mathrm{~ms}$, if necessary, and is $3 \mathrm{~ms}$ long; and the third one occurs at $13 \mathrm{~ms}$, for $2 \mathrm{~ms}$ long. The obtained results with this time slot subdivision, and the parameters presented in Table IX suggest that this communication scheme is reliable.

Fig. 18 shows the aggregate results of the simulations performed, with the average, minimum, and maximum values of reception delays of the followers, from leaders' broadcasted messages, per platoon position. Higher average delay values are presented by the middle platoons, due to the same aforementioned reason. As such, the platoons at the top of the chain present better average delay results, around $5.5 \mathrm{~ms}$, whereas the platoons in the middle present average delay results at about $6.7 \mathrm{~ms}$. Simulation also showed that the first leaders' transmissions were mostly successful. When the corresponding ACK did not arrive in time, a second transmission took place. When the second transmission's ACK was not timely received by the leader, a third transmission occurred. Quantitatively, $89.4 \%$ of the first transmissions were successful; $9.8 \%$ reached their destiny at the second transmission; and only $0.8 \%$ required three transmissions to be properly received.

Since platoon leaders' time slots were augmented by $50 \%$ with respect to previous Matlab/Simulink simulations and the followers' time slots were reduced to half its previous value, a new set of simulations were performed with these different timings. The results are similar to those obtained previously, confirming the appropriate performance of the proposed algorithms.

\section{CONCLUSiON}

A simulation engine for platoons of IVC-enabled autonomous vehicles has been implemented in Matlab/Simulink. Several information-updating schemes have been proposed and assessed through simulation scenarios. The simulation results suggest that the proposed information-updating algorithms are appropriate to the problem under study.

To assess whether DSRC could cope with the tight time constraints of the simulation models, a set of simulations has been performed with the NS-3. The results suggest that the proposed algorithms may operate using the present technology, although some concerns remain with respect to broadcast performance of the DSRC protocol. Moreover, the selected Log Distance signal propagation model used in the NS-3 simulator may be simplistic. Other models (e.g., Three Log Distance or Nakagami with different $m$-parameter values) might probably present less favorable results with respect to leaders' broadcasting since the reliability of this protocol is still an open issue in the research community. Thus, more demanding network scenarios with more complex mobility patterns should also be assessed.

Nevertheless, when using the information-updating Scheme IV proposed and simulated in Sections VI-D and VII-D, respectively, the leader's anticipatory information is already incorporated in the information that each vehicle receives from its precedent and transmits to its own follower, allowing a safe operation with parameter $C_{1}=0$. As such, an all-unicast intraplatooning transmission scheme is feasible, leaving broadcasting for leaders to communicate with other platoons and infrastructure. Moreover, unicast messages are resilient and timely delivered under the simulated scenarios.

Since constant spacing platooning may enable a considerable increase in traffic flow, more simulation results of IVC-enabled autonomous vehicles are required, aiming at the comprehension of new possible representations of the fundamental diagram of traffic flow applied to such scenarios.

Considering the favorable performance presented by the proposed information-updating algorithms in the very demanding scenario of constant spacing platoons, they might also allow important safety and efficiency improvements when applied to different scenarios involving other type of IVC-enabled vehicles.

\section{REFERENCES}

[1] D. Swaroop, "String stability of interconnected systems: An application to platooning in automated highway systems," Ph.D. dissertation, Univ. California, Berkeley, CA, 1994.

[2] D. Swaroop and J. K. Hedrick, "String stability with a constant spacing platooning strategy in automated vehicle following systems," ASME J. Dyn. Syst. Meas. Control, vol. 121, pp. 462-470, Sep. 1999.

[3] R. Rajamani, Vehicle Dynamics and Control. New York: SpringerVerlag, 2006

[4] J. E. Anderson, An Intelligent Transportation Network System: Rationale, Attributes, Status, Economics, Benefits, and Courses of Study for Engineers and Planners. Minneapolis, MN: PRT, 2009

[5] IEEE Standard for Local and Metropolitan Area Networks - Specific requirements Part 11: Wireless LAN Medium Access Control (MAC) and Physical Layer (PHY) Specifications Amendment 6: Wireless Access in Vehicular Environments, IEEE Std. 802.11p-2010, Jul. 2010.

[6] Wireless Access in Vehicular Environments (WAVE)-Networking Services, IEEE Std. 1609.3/D8.0-2010, Jun. 2010.

[7] Wireless Access in Vehicular Environments (WAVE)-Multi-channel Operation, IEEE Std. 1609.4-2010, Dec. 2010.

[8] Long-Term Evolution (LTE)-Advanced. [Online]. Available: http://www.3gpp.org/LTE-Advanced

[9] A. Kesting, M. Treiber, and D. Helbing, "Connectivity statistics of storeand-forward intervehicle communication," IEEE Trans. Intell. Transp. Syst., vol. 11, no. 1, pp. 172-181, Mar. 2010.

[10] M. Parent, "Advanced urban transport: Automation is on the way," IEEE Intell. Syst., vol. 22, no. 2, pp. 9-11, Mar./Apr. 2007.

[11] NS-3, Network Simulator. [Online]. Available: http://www.nsnam.org/

[12] S. Mahal, "Effects on communication delays on string stability in a AHS environment," M.S. thesis, Univ. California, Berkeley, CA, Mar. 2000. 
[13] P. Fernandes and U. Nunes, "Algorithms for management of a multiplatooning system of IVC-enabled autonomous vehicles, with high traffic capacity," in Proc. 14th Int. IEEE Conf. Intell. Transp. Syst., Washington, DC, Oct. 5-7, 2011, pp. 1935-1941.

[14] P. Fernandes and U. Nunes, Platooning with IVC-enabled autonomous vehicles: Inter- and intra-platoon positioning management and cooperative behavior algorithms for high traffic capacity, submitted for publication.

[15] V. Milanés, J. Alonso, L. Bouraoui, and J. Ploeg, "Cooperative maneuvering in close environments among cybercars and dual-mode cars," IEEE Trans. Intell. Transp. Syst., vol. 12, no. 1, pp. 15-24, Mar. 2011.

[16] L. Xiao and F. Gao, "Practical string stability of platoon of adaptive cruise control vehicles," IEEE Trans. Intell. Transp. Syst., vol. 12, no. 4, pp. 1184-1194, Dec. 2011.

[17] C.-L. Huang, Y. Fallah, R. Sengupta, and H. Krishnan, "Intervehicle transmission rate control for cooperative active safety system," IEEE Trans. Intell. Transp. Syst., vol. 12, no. 3, pp. 645-658, Sep. 2011.

[18] S. Shladover, "PATH at 20-History and major milestones," IEEE Trans. Intell. Transp. Syst., vol. 8, no. 4, pp. 584-592, Dec. 2007.

[19] P. Varaiya, "Smart cars on smart roads: Problems of control," IEEE Trans. Autom. Control, vol. AC-38, no. 2, pp. 195-207, Feb. 1993.

[20] L. Alvarez and R. Horowitz, "Safe platooning in automated highway systems," Univ. California, Berkeley, CA, 1997.

[21] R. Horowitz and P. Varaiya, "Control design of an automated highway system," Proc. IEEE-Special Issue Hybrid System, vol. 88, no. 7, pp. 913-925, Jul. 2000.

[22] R. Rajamani, H. S. Tan, B. Law, and W. B. Zhang, "Demonstration of integrated lateral and longitudinal control for the operation of automated vehicles in platoons," IEEE Trans. Control Syst. Technol., vol. 8, no. 4, pp. 695-708, Jul. 2000.

[23] S. Kato, S. Tsugawa, K. Tokuda, T. Matsui, and H. Fujii, "Vehicle control algorithms for cooperative driving with automated vehicles and intervehicle communications," IEEE Trans. Intell. Transp. Syst., vol. 3, no. 3, pp. 155-161, Sep. 2002.

[24] J. Bom, B. Thuilot, F. Marmoiton, and P. Martinet, "A global control strategy for urban vehicular platooning relying on nonlinear decoupling laws," in Proc. IEEE/RSJ Int. Conf. Intell. Robots Syst., Aug. 2005, pp. $2875-2880$.

[25] A. Broggi and M. Lauer, "VisLab's vehicles just reached Siberia: Driverless! Now Kazakhstan and then China until Shanghai," IEEE Intell. Transp. Syst. Mag., vol. 2, no. 3, pp. 43-44, Fall 2010.

[26] X. Liu and A. Goldsmith, "Effects on communication delay on string stability in vehicle platoons," in Proc. IEEE 4th Int. Conf. Intell. Transp. Syst., Oakland, CA, 2001, pp. 625-630.

[27] ULTra PRT. [Online]. Available: http://www.ultraprt.com/prt/

[28] Hermes. [Online]. Available: http://students.ceid.upatras.gr/ xithalis/

[29] Innovative transportation simulator (iTS). [Online]. Available: http://www.maitint.org/

[30] P. Fernandes and U. Nunes, "Platooning of autonomous vehicles with intervehicle communications in SUMO traffic simulator," in Proc. 13th Int. IEEE Annu. Conf. Intell. Transp. Syst., Madeira Island, Portugal, Sep. 19-22, 2010, pp. 1313-1318.

[31] D. Krajzewicz, M. Bonert, and P. Wagner, "The open source traffic simulation package SUMO," in Proc. RoboCup Infrastruct. Simul. Competition, Bremen, Germany, 2006, pp. 1-5.

[32] J. Dold and O. Stursberg, "Distributed predictive control of communicating and platooning vehicles," in Proc. 48th Joint IEEE Conf. Decision Control, 28th Chinese Control Conf., Shanghai, China, Dec. 16-18, 2009, pp. 561-566.

[33] D. Wang and M. Pham, "A high-fidelity co-simulation platform for motion and control research for vehicle platooning," Int. J. Veh. Auton. Syst., vol. 6, no. 1/2, pp. 104-121, 2008.

[34] F. Yu and S. Biswas, "Self-configuring TDMA protocols for enhancing vehicle safety with DSRC based vehicle-to-vehicle communications," IEEE J. Sel. Areas Commun., vol. 25, no. 8, pp. 1526-1537, Oct. 2007.

[35] M. Torrent-Moreno, J. Mittag, P. Santi, and H. Hartenstein, "Vehicleto-vehicle communication: Fair transmit power control for safety-critical information," IEEE Trans. Veh. Technol., vol. 58, no. 7, pp. 3684-3703, Sep. 2009.

[36] Y. Bi, L. Cai, X. Shen, and H. Zhao, "Efficient and reliable broadcast in intervehicle communication networks: A cross-layer approach," IEEE Trans. Veh. Technol., vol. 59, no. 5, pp. 2404-2417, Jun. 2010.
[37] C. E. Palazzi, M. Rosseti, and S. Ferreti, "An intervehicular communication architecture for safety and entertainment," IEEE Trans. Intell. Transp. Syst., vol. 11, no. 1, pp. 90-99, Mar. 2010.

[38] S. Tabatabaei, M. Fleury, N. Qadri, and M. Ghanbari, "Improving propagation modeling in urban environments for vehicular ad hoc networks," IEEE Trans. Intell. Transp. Syst., vol. 12, no. 3, pp. 705-716, Sep. 2011.

[39] A. Kesting, "Microscopic modeling of human and automated driving: Towards traffic-adaptive cruise control," Ph.D. dissertation, VDM Verlag, Saarbrücken, Germany, Feb, 2008.

[40] M. Treiber, A. Hennecke, and D. Helbing, "Congested traffic states in empirical observation and microscopic simulations," Phys. Rev. E, vol. 62 , no. 2, pp. 1805-1824, Aug. 2000.

[41] S. Krauß, "Microscopic modeling of traffic flow: Investigation of collision free vehicle dynamics," Ph.D. dissertation, Köln Univ., Köln, Germany, Apr., 1998.

[42] D. Gazis, Traffic Theory, Kluwer's International Series on Operation Research and Management Science. New York: Springer-Verlag, 2002.

[43] MATLAB/Simulink. [Online]. Available: http://www.mathworks.com

[44] Y. Morgan, "Notes on DSRC and WAVE standards suite, its architecture, design, and characteristics," IEEE J. Commun. Surv. Tutorials, vol. 12, no. 4, pp. 504-518, Sep. 2010.

[45] H. Hartenstein and K. Laberteaux, VANET Vehicular Applications and Inter-Networking Technologies. Chichester, U.K.: Wiley, 2010.

[46] T. Bingmann, "Accuracy enhancements of the 802.11 model and EDCA QoS extensions in ns-3," M.S. thesis, Karlsruhe Univ., Karlsruhe, Germany, 2009.

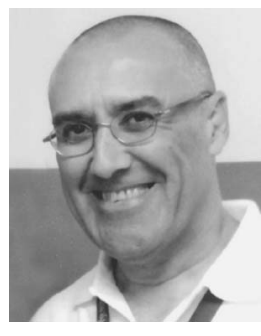

Pedro Fernandes (S'07-M'11) received the Lic. degree in electrical engineering from the University of Coimbra, Coimbra, Portugal. He is currently working toward the Ph.D. degree with the Institute of Systems and Robotics, Department of Electrical and Computer Engineering, University of Coimbra.

Since 2007, he has been participating in several projects. His research interests include intervehicle communications, information management, autonomous vehicles, platooning, and simulation methods applied to intelligent transportation systems.

Mr. Fernandes is member of the Association for Computing Machinery, the Society of Automotive Engineers, the IEEE Communications Society, the IEEE Intelligent Transportation Systems Society, the IEEE Robotics and Automation Society, and the IEEE Vehicular Technology Society. He served as a member of the International Program Committee of the 2007 IEEE International Conference on Vehicular Electronics and Safety.

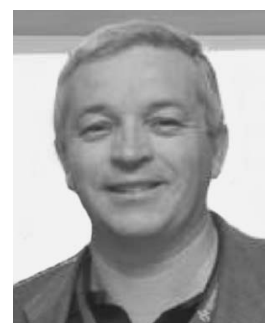

Urbano Nunes (S'90-M'95-SM'09) received the Lic. and $\mathrm{Ph} . \mathrm{D}$. degrees in electrical engineering from the University of Coimbra, Coimbra, Portugal, in 1983 and 1995, respectively.

$\mathrm{He}$ is currently a Full Professor with the Department of Computer and Electrical Engineering, University of Coimbra. He is also the Vice Director of the Coimbra pole of the Institute for Systems and Robotics, where he is the coordinator of the Automation and Mobile Robotics Group. He has been involved with or is responsible for several funded projects at both the national and international levels in the areas of mobile robotics, intelligent vehicles, and intelligent transportation systems (ITS). He has research interests in several areas in connection with intelligent vehicles and human-centered mobile robotics with more than 120 published papers in these areas.

Dr. Nunes is currently the Vice President for Technical Activities of the IEEE ITS Society and an Associate Editor of the IEEE TRANSACTIONS ON INTELLIGENT TRANSPORTATION SYSTEMS and IEEE Intelligent Transportation Systems Magazine. He was the General Chair of the 2010 IEEE Intelligent Transportation Systems Conference, Madeira Islands, Portugal, and he is a General Co-Chair of the 2012 IEEE Conference on Intelligent Robots and Systems. 